\title{
ARITHMETIC DEGREES FOR DYNAMICAL SYSTEMS OVER FUNCTION FIELDS OF CHARACTERISTIC ZERO
}

\author{
YOHSUKE MATSUZAWA, KAORU SANO, AND TAKAHIRO SHIBATA
}

\begin{abstract}
We study arithmetic degree of a dominant rational self-map on a smooth projective variety over a function field of characteristic zero. We see that the notion of arithmetic degree and some related problems over function fields are interpreted into geometric ones. We give another proof of the theorem that the arithmetic degree at any point is smaller than or equal to the dynamical degree. We give a sufficient condition for an arithmetic degree to coincide with the dynamical degree, and prove that any self-map has so many points whose arithmetic degrees are equal to the dynamical degree. We study dominant rational self-maps on projective spaces in detail.
\end{abstract}

\section{Contents}

1. Introduction 1

2. Height functions for varieties over function fields 4

3. Arithmetic degrees for dynamical systems over function fields

4. A fundamental inequality 12

5. A sufficient condition 17

6. Arithmetic degrees for projective spaces 18

7. Construction of orbits 21

References

\section{INTRODUCTION}

Let $K$ be a field where heights $h_{X}$ on smooth projective varieties $X$ can be defined (e.g. a number field or a function field). Given a

2010 Mathematics Subject Classification. Primary 37P15, Secondary 14E05.

Key words and phrases. dynamical systems, arithmetic degree, dynamical degree. 
dominant rational self-map $f: X \rightarrow X$ on a smooth projective variety $X$ and a rational point $P \in X(K)$, it is important to study how the height $h_{X}\left(f^{m}(P)\right)$ varies as $m$ grows. As a quantity representing the growth rate of $h_{X}\left(f^{m}(P)\right)$, Kawaguchi and Silverman defined arithmetic degree of $f$ at $P$ :

$$
\alpha_{f}(P)=\lim _{m \rightarrow \infty} \max \left\{h_{X}\left(f^{m}(P)\right), 1\right\}^{1 / m}
$$

(cf. KaSi2 or Definition 3.2 (ii)). On the other hand, there is another invariant for self-maps, the (first) dynamical degree of $f$ :

$$
\delta_{f}=\lim _{m \rightarrow \infty}\left(\left(f^{m}\right)^{*} H \cdot H^{\operatorname{dim} X-1}\right)^{1 / m},
$$

where $H$ is an ample Cartier divisor on $X$ (cf. Definition 3.2 (i)). It seems important and interesting to investigate relations between those two quantities. Especially, it is natural to ask when $\alpha_{f}(P)$ coincides with $\delta_{f}$. Kawaguchi and Silverman conjectured some properties of arithmetic degree and a sufficient condition for a rational point $P$ on which the arithmetic degree at $P$ coincides with the dynamical degree (cf. KaSi2, Conjecture 6]). These conjectures have been verified in some special cases. For details, see Conjecture 3.4, Remark 3.5] and Remark 3.6.

In this article, we study arithmetic degrees over function fields in one variable over an algebraically closed field of characteristic zero. The advantage of considering height theory over function fields is that we can interpret the height of a rational point into the degree of the curve corresponding to the rational point. A variety $X$ over a function field $K$ of a curve $C$ can be seen as the generic fiber of a fibration $\pi: \mathcal{X} \rightarrow C$ over $C$, and then a $K$-rational point $P$ of $X$ corresponds to a section $\sigma$ of $\pi$. In this situation, the height $h_{X}(P)$ of $P$ is equal to $\operatorname{deg}\left(\sigma^{*} \mathcal{H}\right)$, where $\mathcal{H}$ is a $\pi$-ample Cartier divisor on $\mathcal{X}$. So arithmetic degree is also described by the degrees of divisors. We will use this geometric interpretation to deduce the results in this article.

A fundamental relation on those two types of degree is the following inequality:

$$
\alpha_{f}(P) \leq \delta_{f}
$$

This inequality was proved over any field where height functions can be defined by Kawaguchi-Silverman and Matsuzawa (cf. [KaSi2, Theorem 4] and [Mat]). We will prove it over function fields of characteristic 0 by using the geometric interpretation of height (Theorem 4.1). Our proof works only over function fields, but it seems simple and short.

After we obtain the fundamental inequality, it is natural to ask when the arithmetic degree of a given rational point attains the dynamical 
degree. Over $\overline{\mathbb{Q}}$, Kawaguchi and Silverman predicts a sufficient condition for a rational point to have the arithmetic degree which is equal to the dynamical degree (Conjecture 3.4 (iv)). However, over $\overline{k(t)}$, this conjecture does not hold in general (Example 3.8). We give a sufficient condition for the arithmetic degree of a rational point to attain the dynamical degree as a geometric condition of the corresponding section (Theorem 5.1). For a dynamical system on a projective space, we will give some other sufficient conditions (Theorem 6.2 and Theorem 6.4).

It is also important to investigate whether there exists a rational point whose arithmetic degree attains the dynamical degree. Over $\overline{k(t)}$ with $k$ an uncountable algebraically closed field of characteristic 0 , we will prove the result that there are densely many points having pairwise disjoint orbits such that the arithmetic degree of them attain the dynamical degree (Theorem 7.2 ). Over number fields, this theorem has been proved only for some particular cases (cf. [KaSi1, Theorem 3] and [MSS, Theorem 1.7]). More strongly, for a self-map on a smooth projective rational variety, we can take such points over a fixed function field (Theorem 7.5 ).

\section{Notation.}

- Throughout this article, $k$ denotes an algebraically closed field of characteristic zero, and $\overline{k(t)}$ denotes the algebraic closure of the rational function field of one variable over $k$.

- For a rational map $f: X \rightarrow Y, I_{f}$ denotes the indeterminacy locus of $f$.

- A curve simply means a smooth projective variety of dimension 1 unless otherwise stated.

- For any $\mathbb{R}$-valued function $h(x)$, we set $h^{+}(x)=\max \{h(x), 1\}$.

- Let $f, g$ and $h$ be $\mathbb{R}$-valued functions on a domain $S$. The equality $f=g+O(h)$ means that there is a positive constant $C$ such that $|f(x)-g(x)| \leq C|h(x)|$ for every $x \in S$. The equality $f=g+O(1)$ means that there is a positive constant $C^{\prime}$ such that $|f(x)-g(x)| \leq C^{\prime}$ for every $x \in S$.

Acknowledgments. The authors would like to thank Professors Osamu Fujino and Shu Kawaguchi for reading a manuscript of this paper and giving useful comments. The first author wold like to thank Professor Tomohide Terasoma for attending his seminar and giving valuable comments. 


\section{HEIGHT FUNCTIONS FOR VARIETIES OVER FUNCTION FIELDS}

In this section, we define the (Weil) height functions on projective varieties over $\overline{k(t)}$, and see that there is another description of height in terms of the degree of a divisor on a curve. Basic facts of (Weil) height functions over function fields is explained for example in Lan, Chapter 3, §3] and [HiSi, B.10]. So we omit most of the proofs.

First, we define the height functions on projective spaces over function fields.

Definition 2.1. Let $C$ be a (smooth projective) curve over $k$ and $p \in C$ a closed point. We define a valuation $v_{p}: K(C)^{\times} \rightarrow \mathbb{Z}$ as

$$
v_{p}(f)=(\text { the multiplicity of } f \text { at } p) \text {. }
$$

This is nothing but the discrete valuation associated to the discrete valuation ring $\mathcal{O}_{C, p}$. Set $v_{p}(0)=\infty$.

Definition 2.2. Let $C$ be a curve over $k$. Take $P \in \mathbb{P}^{n}(K(C))$. Represent $P$ by homogeneous coordinates as $P=\left(f_{0}: f_{1}: \cdots: f_{n}\right)$, where $f_{i} \in K(C)$. We define the height function on $\mathbb{P}^{n}(K(C))$ relative to $K(C)$ as

$$
h_{K(C)}(P)=\sum_{p \in C}-\min \left\{v_{p}\left(f_{0}\right), \ldots, v_{p}\left(f_{n}\right)\right\} .
$$

Remark 2.3. (i) Since $\#\left\{p \in C \mid v_{p}(f) \neq 0\right\}$ is finite for any $f \in$ $K(C)^{\times}, \sum_{p \in C}-\min \left\{v_{p}\left(f_{0}\right), \ldots, v_{p}\left(f_{n}\right)\right\}$ is in fact a finite sum.

(ii) $h_{K(C)}(P)$ is independent of the representation of $P$. For, if we represent $P$ as $P=\left(g f_{0}: \cdots: g f_{n}\right)$, where $g \in K(C)^{\times}$, then

$$
\begin{aligned}
\sum_{p}-\min _{i} v_{p}\left(g f_{i}\right) & =\sum_{p}-\min _{i}\left(v_{p}(g)+v_{p}\left(f_{i}\right)\right) \\
& =\sum_{p}-v_{p}(g)+\sum_{p}-\min v_{p}\left(f_{i}\right) \\
& =\sum_{p}-\min _{i} v_{p}\left(f_{i}\right)
\end{aligned}
$$

since $\sum_{p}-v_{p}(g)=-\operatorname{deg}((g))=0$.

Next, we give the definition of the (absolute) height function on $\mathbb{P}^{n}(\overline{k(t)})$ which is essentially compatible with the previous definition of height functions over function fields.

Lemma 2.4 (cf. [HiSi, Lemma B.2.1]). Let $\phi: C^{\prime} \rightarrow C$ be a finite surjective morphism of two curves. Set $K=K(C), K^{\prime}=K\left(C^{\prime}\right)$. Take 
$P \in \mathbb{P}^{n}(K)$. Then

$$
\frac{1}{[K: k(t)]} h_{K}(P)=\frac{1}{\left[K^{\prime}: k(t)\right]} h_{K^{\prime}}(P) .
$$

We define the (absolute) height function on $\mathbb{P}^{n}(\overline{k(t)})$ as follows:

Definition 2.5. Take $P \in \mathbb{P}^{n}(\overline{k(t)})$. We define the height function on $\mathbb{P}^{n}(\overline{k(t)})$ as

$$
h(P)=\frac{1}{[K: k(t)]} h_{K}(P),
$$

where $K$ is a finite extension field of $k(t)$ such that $P \in \mathbb{P}^{n}(K) . h(P)$ is independent of the choice of $K$ by Lemma 2.4 .

Using the height functions on projective spaces, we define the height function on a projective variety $X$ associated to a Cartier divisor. We prepare the following lemma.

Lemma 2.6 (cf. [HiSi, Proposition B.2.4 and Theorem B.3.1]). Let $X$ be a projective variety over $\overline{k(t)}$ and $D$ a base point free Cartier divisor on $X$.

(i) Let $\phi: X \rightarrow \mathbb{P}^{n}, \psi: X \rightarrow \mathbb{P}^{n}$ be two morphisms associated to the complete linear system $|D|$. Then $h \circ \phi=h \circ \psi+O(1)$.

(ii) Let $\phi_{1}: X \rightarrow \mathbb{P}^{n_{1}}, \phi_{2}: X \rightarrow \mathbb{P}^{n_{2}}$ be morphisms associated to very ample Cartier divisors $A_{1}, A_{2}$ on $X$, respectively. Let $\phi: X \rightarrow \mathbb{P}^{n}$ be a morphism associated to $A_{1}+A_{2}$. Then $h \circ \phi=$ $h \circ \phi_{1}+h \circ \phi_{2}+O(1)$.

Using Lemma 2.6, we can define the height function associated to a Cartier divisor up to the difference of a bounded function.

Definition 2.7. Let $X$ be a projective variety over $\overline{k(t)}$.

(i) Let $A$ be a base point free Cartier divisor on $X$. We define a height function on $X$ associated to $A$ as

$$
h_{A}=h \circ \phi_{A},
$$

where $\phi_{A}$ is a morphism associated to $|A|$. By Lemma 2.6 (i), $h_{A}$ is well-defined up to a bounded function.

(ii) Let $D$ be a Cartier divisor on $X$. We define a height function on $X$ associated to $D$ as

$$
h_{D}=h \circ \phi_{A}-h \circ \phi_{B},
$$

where $A, B$ are base point free Cartier divisors such that $D \sim A-B$ and $\phi_{A}, \phi_{B}$ are morphisms associated to $|A|,|B|$, respectively. 
Note that we can always take such $A$ and $B$. Assume that we have other base point free Cartier divisors $A^{\prime}, B^{\prime}$ such that $D \sim A^{\prime}-B^{\prime}$. Then $A+B^{\prime} \sim A^{\prime}+B$. Lemma 2.6 (i) implies that $h_{A+B^{\prime}}=h_{A^{\prime}+B}+$ $O(1)$. Moreover, by Lemma 2.6 (ii), $h_{A+B^{\prime}}=h_{A}+h_{B^{\prime}}+O(1)$ and $h_{A^{\prime}+B}=h_{A^{\prime}}+h_{B}+O(1)$. So $h_{A}-h_{B}=h_{A^{\prime}}-h_{B^{\prime}}+O(1)$. Hence $h_{D}$ is well-defined up to a bounded function.

Proposition 2.8 (cf. [HiSi, Theorem B.3.2]). Let $X$ be a projective variety over $\overline{k(t)}$.

(i) Let $D, D^{\prime}$ be Cartier divisors on $X$ and $n, n^{\prime}$ be integers. Then

$$
h_{n D+n^{\prime} D^{\prime}}=n h_{D}+n^{\prime} h_{D^{\prime}}+O(1) .
$$

(ii) Let $f: X \rightarrow Y$ be a morphism to a projective variety $Y$ and $E$ a Cartier divisor on $Y$. Then

$$
h_{f^{*} E}=h_{E} \circ f+O(1) .
$$

Next, we give another description of height in terms of the degree of a divisor on a curve.

Lemma 2.9. Let $X, S$ be integral schemes and $f: X \rightarrow S$ a morphism of finite type. Let $\eta$ be the generic point of $S$ and $X_{\eta}$ the generic fiber of $f$. For a rational section $\sigma: S \rightarrow X$ of $f$ (i.e. a rational map $\sigma: S \rightarrow X$ such that $\left.f \circ \sigma=\mathrm{id}_{S}\right), P \in X_{\eta}(k(\eta))$ denotes the rational point obtained by the base change of $\sigma$ along $k(\eta) \rightarrow S$. Then the mapping $\sigma \mapsto P$ gives a one-to-one correspondence between the set of rational sections of $f$ and $X_{\eta}(k(\eta))$.

This lemma follows from an elementary scheme-theoretic argument. When $X$ is a projective variety and $S=C$ is a (smooth projective) curve, Lemma 2.9 is reduced to the following.

Proposition 2.10. Let $C$ be a curve over $k$ and set $K=K(C)$.

(i) Let $\pi: X \rightarrow C$ be a surjective morphism from a projective variety $X$ to $C$ and $X_{\eta}$ the generic fiber of $\pi$. Then $X_{\eta}(K)$ corresponds one-to-one to the set of sections of $\pi$.

(ii) Let $Y_{k}$ be a projective variety over $k$ and set $Y_{K}=Y_{k} \times_{k} K$. Then $Y_{K}(K)$ corresponds one-to-one to the set of $k$-morphisms from $C$ to $Y_{k}$.

Proof. (i) Note that any rational map $\varphi: C \rightarrow X$ is in fact a morphism since codim $I_{\varphi} \geq 2$. So the assertion follows from Lemma 2.9.

(ii) Apply (i) to the projection $\operatorname{pr}_{C}: Y_{k} \times_{k} C \rightarrow C$. Note that the sections of $\mathrm{pr}_{C}$ correspond one-to-one to the $k$-morphisms from $C$ to $Y_{k}$. 
Here is another description of height by the degree of divisors for projective spaces.

Proposition 2.11 (cf. [HiSi, Lemma B.10.1]). Let $C$ be a curve and set $K=K(C)$. Take $P \in \mathbb{P}^{n}(K)$ and let $g: C \rightarrow \mathbb{P}_{k}^{n}$ be the corresponding morphism. Then

$$
h(P)=\frac{1}{[K: k(t)]} \operatorname{deg}\left(g^{*} \mathcal{O}(1)\right) .
$$

Proof. For $P=\left(f_{0}: \cdots: f_{n}\right) \in \mathbb{P}^{n}(K), g$ is represented as $g(x)=$ $\left(f_{0}(x): \cdots: f_{n}(x)\right)$. Let $H_{i}=\left(x_{i}\right)$ be the hyperplane of $\mathbb{P}_{k}^{n}$ associated to the $i$-th coordinate. Assume that $g(C) \subset H_{i}$ for some $i$. Then $f_{i}=0$, so

$$
h_{K}(P)=\sum_{p \in C}-\min _{j \neq i} v_{p}\left(f_{j}\right) .
$$

On the other hand, $\operatorname{deg}\left(g^{*} \mathcal{O}_{\mathbb{P}^{n}}(1)\right)=\operatorname{deg}\left(g^{*} \mathcal{O}_{H_{i}}(1)\right)$. Therefore we can replace $\mathbb{P}_{k}^{n}$ by $H_{i} \cong \mathbb{P}_{k}^{n-1}$. If $g$ is a constant mapping, then $f_{i} \in k$ for all $i$ and $h_{K}(P)=\operatorname{deg}\left(g^{*} \mathcal{O}(1)\right)=0$. So we may assume that $g(C)$ is not contained $H_{i}$ for all $i$.

Set $U=\operatorname{Spec} A=C \backslash g^{-1}\left(H_{0}\right)$ and $f_{i}=\left(\left.g\right|_{U}\right)^{*}\left(x_{i} / x_{0}\right) \in A$. Then $P=\left(1: f_{1}: \cdots: f_{n}\right)$. So

$$
\begin{aligned}
h_{K}(P) & =\sum_{p \in C}-\min \left\{v_{p}(1), v_{p}\left(f_{1}\right), \ldots, v_{p}\left(f_{n}\right)\right\} \\
& =\sum_{p \in C \backslash U}-\min \left\{v_{p}(1), v_{p}\left(f_{1}\right), \ldots, v_{p}\left(f_{n}\right)\right\} \\
& =\sum_{p \in C \backslash U}-\min _{1 \leq i \leq n} v_{p}\left(f_{i}\right) \\
& =\sum_{p \in C \backslash U}-\min _{1 \leq i \leq n} v_{p}\left(g^{*}\left(x_{i} / x_{0}\right)\right) \\
& =\sum_{p \in C \backslash U}-\min _{1 \leq i \leq n} v_{p}\left(g^{*} H_{i}\right)+\sum_{p \in C \backslash U} v_{p}\left(g^{*} H_{0}\right),
\end{aligned}
$$

where the second equality holds because $v_{p}\left(f_{i}\right) \geq 0$ for $p \in U$ and the third equality holds because $\min _{1 \leq i \leq n} v_{p}\left(f_{i}\right)<0$ for $p \in C \backslash U$. Obviously

$$
\sum_{p \in C \backslash U} v_{p}\left(g^{*} H_{0}\right)=\sum_{p \in C} v_{p}\left(g^{*} H_{0}\right)=\operatorname{deg}\left(g^{*} H_{0}\right) .
$$

For $p \in C \backslash U, v_{p}\left(g^{*} H_{i}\right)>0$ is equivalent to $g(p) \in H_{i}$. Since $g(p) \in H_{0}, g(p) \notin H_{i}$ for some $i>0$ and then $v_{p}\left(g^{*} H_{i}\right)=0$. Hence 
$\min _{1 \leq i \leq n} v_{p}\left(g^{*} H_{i}\right)=0$ for all $p \in C \backslash U$. As a consequence,

$$
h(P)=\frac{1}{[K: k(t)]} \operatorname{deg}\left(g^{*} H_{0}\right)=\frac{1}{[K: k(t)]} \operatorname{deg}\left(g^{*} \mathcal{O}(1)\right) .
$$

By Proposition 2.11, we can see that $h(P) \geq 0$ for any $P \in \mathbb{P}^{n}(K)$. Furthermore, for a rational point $P \in \mathbb{P}^{n}(K)$ corresponding to a morphism $g: C \rightarrow \mathbb{P}_{k}^{n}, P \in \mathbb{P}^{n}(k)$ if and only if $g$ is a constant map. So we obtain the following.

\section{Proposition 2.12.}

(i) $h(P) \geq 0$ for any $P \in \mathbb{P}^{n}(\overline{k(t)})$.

(ii) For $P \in \mathbb{P}^{n}(\overline{k(t)}), h(P)=0$ if and only if $P \in \mathbb{P}^{n}(k)$.

We give a description of height by the degree of divisors for a projective variety over $\overline{k(t)}$ which has a model over a curve or, more strongly, over $k$.

Definition 2.13. Let $X$ be a projective variety over $\overline{k(t)}$ and $H$ an ample Cartier divisor on $X$. We define a function $\tilde{h}_{H}: X(\overline{k(t)}) \rightarrow \mathbb{R}_{\geq 0}$ as follows. Fix a model $\left(X_{C} \stackrel{\pi}{\rightarrow} C, H_{C}\right)$ of $(X, H)$ over a curve $C$, that is, a projective variety $X_{C}$ over $k$ with a surjection $\pi: X_{C} \rightarrow C$ whose geometric generic fiber is $X$, and a $\pi$-ample Cartier divisor $H_{C}$ on $X_{C}$ such that $\left(X \rightarrow X_{C}\right)^{*} H_{C} \sim H$. For any $P \in X(\overline{k(t)})$, take a curve $C_{1}$ with $K\left(C_{1}\right) \supset K(C)$ and the section $\sigma_{1}$ of $\pi_{C_{1}}: X_{C} \times_{C} C_{1} \rightarrow C_{1}$ corresponding to $P$, and set $H_{C_{1}}=\left(X_{C} \times_{C} C_{1} \rightarrow X_{C}\right)^{*} H_{C}$ and

$$
\tilde{h}_{H}(P)=\frac{1}{\left[K\left(C_{1}\right): k(t)\right]} \operatorname{deg}\left(\sigma_{1}^{*} H_{C_{1}}\right) \text {. }
$$

Proposition 2.14. Notation is as in Definition 2.13. Then $\tilde{h}_{H}$ is a well-defined height function associated to $H$.

Proof. Take any point $P \in X(\overline{k(t)})$. Take curves $C_{i}$ with $K\left(C_{i}\right) \supset$ $K(C)$ and the sections $\sigma_{i}$ of $\pi_{C_{i}}: X_{C_{i}}=X_{C} \times_{C} C_{i} \rightarrow C_{i}$ for $i=1,2$. To see the well-definedness of $\tilde{h}_{H}$, we may assume that $K\left(C_{2}\right) \supset K\left(C_{1}\right)$.

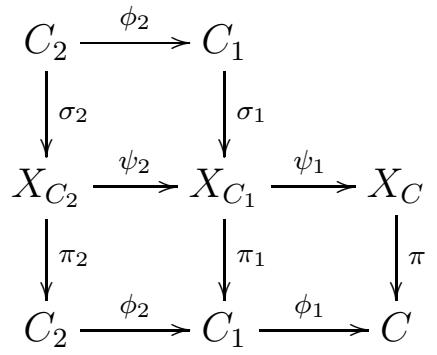


Set $H_{C_{1}}=\psi_{1}^{*} H_{C}$ and $H_{C_{2}}=\psi_{2}^{*} H_{C_{1}}$. Then

$$
\begin{aligned}
\frac{\operatorname{deg}\left(\sigma_{2}^{*} H_{C_{2}}\right)}{\left[K\left(C_{2}\right): k(t)\right]} & =\frac{\operatorname{deg}\left(\phi_{2}^{*} \sigma_{1}^{*} H_{C_{1}}\right)}{\left[K\left(C_{2}\right): K\left(C_{1}\right)\right]\left[K\left(C_{1}\right): k(t)\right]} \\
& =\frac{\operatorname{deg}\left(\sigma_{1}^{*} H_{C_{1}}\right)}{\left[K\left(C_{1}\right): k(t)\right]} .
\end{aligned}
$$

So it follows that $\tilde{h}_{H}(P)$ is well-defined.

Take a sufficiently large integer $N$ such that there is a morphism $\iota_{C}: X_{C} \rightarrow \mathbb{P}_{k}^{n} \times C$ over $C$ with $\iota_{C}^{*} \mathcal{O}_{\mathbb{P}_{C}^{n}}(1) \sim_{\pi} N H_{C}$. Take a Cartier divisor $D_{C}$ on $C$ such that $\iota_{C}^{*} \mathcal{O}_{\mathbb{P}_{C}^{n}}(1) \sim N H_{C}+\pi^{*} D_{C}$. Let $\iota: X \rightarrow \mathbb{P} \frac{n}{k(t)}$ be the base change of $\iota_{C}$ by Spec $\overline{k(t)} \rightarrow C$. Then the function $\frac{1}{N} h \circ \iota$ is a height function associated to $H . \iota(P) \in \mathbb{P}^{n}(\overline{k(t)})$ corresponds to the morphism $\operatorname{pr}_{\mathbb{P}^{n}} \circ \iota_{C_{1}} \circ \sigma_{1}: C_{1} \rightarrow \mathbb{P}_{k}^{n}$, where $\iota_{C}$ be the base change of $\iota_{C}$ by $C_{1} \rightarrow C$. We compute

$$
\begin{aligned}
\frac{1}{N} h(\iota(P)) & =\frac{\operatorname{deg}\left(\left(\operatorname{pr}_{\mathbb{P}_{k}^{n}} \circ \iota_{C_{1}} \circ \sigma_{1}\right)^{*} \mathcal{O}_{\mathbb{P}_{k}^{n}}(1)\right)}{N\left[K\left(C_{1}\right): k(t)\right]} \quad \text { (by Proposition 2.11) } \\
& =\frac{\operatorname{deg}\left(\sigma_{1}^{*}\left(N H_{C_{1}}+\psi_{1}^{*} \pi^{*} D_{C}\right)\right)}{N\left[K\left(C_{1}\right): k(t)\right]} \\
& =\frac{\operatorname{deg}\left(\sigma_{1}^{*}\left(N H_{C_{1}}\right)+\left[K\left(C_{1}\right): K(C)\right] \operatorname{deg}\left(D_{C}\right)\right.}{N\left[K\left(C_{1}\right): k(t)\right]} \\
& =\tilde{h}_{H}(P)+\frac{\operatorname{deg}\left(D_{C}\right)}{N[K(C): k(t)]} .
\end{aligned}
$$

So $\tilde{h}_{H}$ is a height function associated to $H$.

\section{ARIthmetic Degrees For DYNAMiCAl SyStems OVER FUNCTION FIELDS}

Definition 3.1. A dynamical system over a field $K$ is a pair $(X, f)$ of a smooth projective variety $X$ over $K$ and a dominant rational self-map $f: X \rightarrow X$ over $K$.

Let $(X, f)$ be a dynamical system over a field $K$. Set

$$
X_{f}=\left\{P \in X(K) \mid f^{m}(P) \notin I_{f} \text { for every } m \geq 0\right\} .
$$

For $P \in X_{f}$, set

$$
O_{f}(P)=\left\{f^{m}(P) \mid m \in \mathbb{Z}_{\geq 0}\right\},
$$

which we call the (forward) $f$-orbit of $P$.

Let $H$ be an ample divisor on $X$. The (first) dynamical degree of $f$ is the number

$$
\delta_{f}=\lim _{m \rightarrow \infty}\left(\left(f^{m}\right)^{*} H \cdot H^{\operatorname{dim} X-1}\right)^{1 / m} \in[1, \infty) .
$$


It is known that the limit converges and $\delta_{f}$ is independent of the choice of $H$.

Definition 3.2. Let $(X, f)$ be a dynamical system over an algebraically closed field $K$ where heights are well-defined (e.g. $\overline{k(t)}$ or $\overline{\mathbb{Q}}$ ). Take an ample Cartier divisor $H$ on $X$ and a rational point $P \in X_{f}$. The arithmetic degree of $f$ at $P$ is defined as

$$
\alpha_{f}(P)=\lim _{m \rightarrow \infty} h_{H}^{+}\left(f^{m}(P)\right)^{1 / m},
$$

where $h_{H}$ is a height function associated to $H$ and $h_{H}^{+}(P)=\max \left\{h_{H}(P), 1\right\}$. Note that we do not know whether the limit converges (cf. Conjecture 3.4 (i)). Similarly, $\bar{\alpha}_{f}(P), \underline{\alpha}_{f}(P)$ are defined as

$$
\begin{aligned}
& \bar{\alpha}_{f}(P)=\limsup _{m \rightarrow \infty} h_{H}^{+}\left(f^{m}(P)\right)^{1 / m}, \\
& \underline{\alpha}_{f}(P)=\liminf _{m \rightarrow \infty} h_{H}^{+}\left(f^{m}(P)\right)^{1 / m} .
\end{aligned}
$$

Proposition 3.3 (cf. [KaSi2, Proposition 14]). In the notation of Definition 3.2. $\alpha_{f}(P), \bar{\alpha}_{f}(P)$ and $\underline{\alpha}_{f}(P)$ are independent of the choices of $H$ and $h_{H}$.

Proof. It is obvious that $\alpha_{f}(P), \bar{\alpha}_{f}(P)$ and $\underline{\alpha}_{f}(P)$ are independent of the choice of $h_{H}$ for a fixed ample Cartier divisor $H$.

Let $H^{\prime}$ be another ample Cartier divisor on $X$. Take a sufficiently large integer $N$ such that $N H-H^{\prime}$ is ample. Then

$$
\begin{aligned}
\lim _{m \rightarrow \infty} h_{H}^{+}\left(f^{m}(P)\right)^{1 / m} & =\lim _{m \rightarrow \infty}\left(\frac{1}{N} h_{N H}^{+}\left(f^{m}(P)\right)\right)^{1 / m} \\
& =\lim _{m \rightarrow \infty} h_{N H}^{+}\left(f^{m}(P)\right)^{1 / m} \\
& =\lim _{m \rightarrow \infty} \max \left\{h_{N H-H^{\prime}}\left(f^{m}(P)\right)+h_{H^{\prime}}\left(f^{m}(P)\right), 1\right\}^{1 / m} \\
& \geq \lim _{m \rightarrow \infty} h_{H^{\prime}}^{+}\left(f^{m}(P)\right)^{1 / m},
\end{aligned}
$$

where we take $h_{N H-H^{\prime}}$ as a non-negative function. Similarly,

$$
\lim _{m \rightarrow \infty} h_{H^{\prime}}^{+}\left(f^{m}(P)\right)^{1 / m} \geq \lim _{m \rightarrow \infty} h_{H}^{+}\left(f^{m}(P)\right)^{1 / m} .
$$

So $\alpha_{f}(P)$ is independent of $H$. The proofs for $\bar{\alpha}_{f}(P)$ and $\underline{\alpha}_{f}(P)$ are similar.

Now we introduce the following conjecture (over $\overline{\mathbb{Q}}$ ) given by Kawaguchi and Silverman (see [KaSi2]).

Conjecture 3.4 (The Kawaguchi-Silverman conjecture over $\overline{\mathbb{Q}}$ ). Let $(X, f)$ be a dynamical system over $\overline{\mathbb{Q}}$. 
(i) The limit defining $\alpha_{f}(P)$ exists for every $P \in X_{f}$.

(ii) $\alpha_{f}(P)$ is an algebraic integer for every $P \in X_{f}$.

(iii) $\left\{\alpha_{f}(P) \mid P \in X_{f}\right\}$ is a finite set.

(iv) Take $P \in X_{f}$. Assume that $O_{f}(P)$ is Zariski dense in $X$. Then $\alpha_{f}(P)=\delta_{f}$.

Remark 3.5. In the case when $f$ is a morphism (see KaSi3, Theorem 2]), Kawaguchi and Silverman showed that (i), (ii), and (iii) of Conjecture 3.4 hold even over global fields of characteristic zero.

Remark 3.6. It is known that Conjecture 3.4 holds in several cases (cf. [MSS, Remark 1.3]).

As the number field case, we consider:

Problem 3.7. Let $(X, f)$ be a dynamical system over $\overline{k(t)}$. Take a point $P \in X_{f}$. When the equality $\alpha_{f}(P)=\delta_{f}$ holds?

The following examples show that the function field case of Conjecture 3.4 (iv) is not true.

Example 3.8. (i) Let $f: \mathbb{P} \frac{1}{k(t)} \rightarrow \mathbb{P} \frac{1}{k(t)}$ be a surjective endomorphism with $\delta_{f}>1$. Take a $k$-valued non-preperiodic point $P \in \mathbb{P}^{1}(k)$. Then $O_{f}(P)$ is Zariski dense in $\mathbb{P} \frac{1}{k(t)}$, but $\alpha_{f}(P)=1<\delta_{f}$.

(ii) Define $f: \mathbb{A} \frac{2}{k(t)} \rightarrow \mathbb{A} \frac{2}{k(t)}$ as $f(x, y)=\left(x^{2}, y^{3}\right)$. Then $f$ naturally extends to the morphism $f: \mathbb{P}_{\overline{k(t)}}^{2} \rightarrow \mathbb{P}_{\overline{k(t)}}^{2}$ and $\delta_{f}=3$. Take a point $P=(t, 2) \in \mathbb{A}^{2}(k(t))$. Then $f^{m}(P)=\left(t^{2^{m}}, 2^{3^{m}}\right)$ and

$$
\alpha_{f}(P)=\lim _{m \rightarrow \infty} \max \left\{\operatorname{deg}\left(t^{2^{m}}\right), \operatorname{deg}\left(2^{3^{m}}\right)\right\}^{1 / m}=\lim _{m \rightarrow \infty}\left(2^{m}\right)^{1 / m}=2 .
$$

We show that $O_{f}(P)=\left\{\left(t^{2^{m}}, 2^{3^{m}}\right)\right\}_{m=0}^{\infty}$ is dense in $\mathbb{P}_{\overline{k(t)}}^{2}$. It is enough to show that $O_{f}(P)$ is dense in $\mathbb{A}_{k(t)}^{2}$. Suppose $O_{f}(P)$ is contained in the zero locus of a polynomial $\phi(t, x, y) \in k(t)[x, y]$. Multiplying $\phi$ with a polynomial in $k[t]$, we may assume that $\phi \in k[t, x, y]$. Set $\phi(t, x, y)=\phi_{r}(t, y) x^{r}+\phi_{r-1} x^{r-1}+\cdots+\phi_{0}(t, y), \phi_{r}(t, y) \neq 0$. Вy assumption, $\phi\left(t, t^{2^{m}}, 2^{3^{m}}\right)=0$ as a polynomial in $k[t]$. Since $\operatorname{deg}\left(t^{2^{m} r}\right)>\operatorname{deg}\left(\phi\left(t, t^{2^{m}}, 2^{3^{m}}\right)-\phi_{r}\left(t, 2^{3^{m}}\right) t^{2^{m} r}\right)=\operatorname{deg}\left(-\phi_{r}\left(t, 2^{3^{m}}\right) t^{2^{m} r}\right)$ for sufficiently large $m$, it follows that $\phi_{r}\left(t, 2^{3^{m}}\right) t^{2^{m} r}=0$ as a polynomial in $k[t]$ for sufficiently large $m$. Therefore $\phi_{r}(t, y)=0$ as a polynomial in $k[t, y]$, which is a contradiction. So $O_{f}(P)$ is Zariski dense in $\mathbb{P}_{k(t)}^{2}$.

In the rest of this article, we will find some other conditions for an arithmetic degree to coincide with the dynamical degree. 


\section{A fundamental inequality}

There is a fundamental inequality between arithmetic degrees and dynamical degrees:

Theorem 4.1 ([KaSi2, Theorem 4] and [Mat]). $K$ denotes an algebraically closed field where heights are well-defined. Let $(X, f)$ be a dynamical system over $K$. Then

$$
\bar{\alpha}_{f}(P) \leq \delta_{f}
$$

holds for any $P \in X_{f}$.

Remark 4.2. This inequality was stated by Kawaguchi and Silverman (see [KaSi2, Theorem 4]), and a correct proof of it was given by Matsuzawa (see [Mat]).

We will give another proof of the inequality over $\overline{k(t)}$.

Theorem 4.3. Let $(X, f)$ be a dynamical system over $\overline{k(t)}$. Then the inequality

holds for any $P \in X_{f}$.

$$
\bar{\alpha}_{f}(P) \leq \delta_{f}
$$

To prove Theorem 4.3, we prepare some lemmas. To begin with, we define a model of a dynamical system over $\overline{k(t)}$.

Definition 4.4. Let $(X, f)$ be a dynamical system over $\overline{k(t)}$. A model of the dynamical system $(X, f)$ over a curve $C$ is a pair $\left(X_{C} \stackrel{\pi}{\rightarrow} C, f\right)$ of a surjective morphism $\pi: X_{C} \rightarrow C$ from a smooth projective $k$-variety $X_{C}$ to $C$ and a dominant rational self-map $f_{C}: X_{C} \rightarrow X_{C}$ over $C$ such that $X_{C} \times_{C} \overline{k(t)}=X$ and the base change of $f_{C}$ along Spec $\overline{k(t)} \rightarrow C$ is equal to $f$.

Lemma 4.5. Let $(X, f)$ be a dynamical system over $\overline{k(t)}$. Then there exists a model of $(X, f)$ over a curve $C$.

Such a model is obtained by resolution of singularities.

Lemma 4.6 (cf. [KaSi2, Proposition 19]). Let $f: X \rightarrow Y$ and $g$ : $Y \rightarrow Z$ be dominant rational maps of smooth projective varieties. Take a Cartier divisor $H$ on $Z$ and a curve $C$ on $X$.

(i) If $C \not \subset I_{f}, f(C) \not \subset I_{g}$ and $H$ is nef, then

$$
\left((g \circ f)^{*} H \cdot C\right) \leq\left(f^{*} g^{*} H \cdot C\right) \text {. }
$$

(ii) If $C \cap I_{f}=\varnothing$ and $f(C) \cap I_{g}=\varnothing$, then

$$
\left((g \circ f)^{*} H \cdot C\right)=\left(f^{*} g^{*} H \cdot C\right) \text {. }
$$


Proof. Since a nef divisor is the limit of a sequence of ample divisors, we may assume that $H$ is ample.

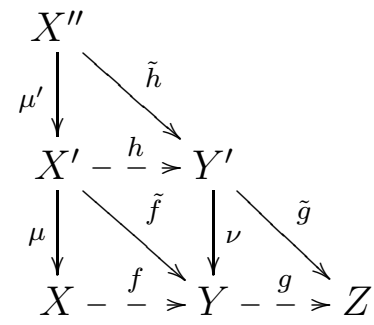

In the above diagram, $\tilde{f}: X^{\prime} \rightarrow Y$ (resp. $\tilde{g}: Y^{\prime} \rightarrow Z$ ) is an elimination of indeterminacy of $f$ (resp. $g$ ) by blowing up smooth centers in $I_{f}$ (resp. $I_{g}$ ), $h=\nu^{-1} \circ f \circ \mu$, and $\tilde{h}: X^{\prime \prime} \rightarrow Y^{\prime}$ is an elimination of indeterminacy of $h$ by blowing up smooth centers in $I_{h}$. Then

$$
\begin{aligned}
& (g \circ f)^{*} H \cdot C \leq f^{*} g^{*} H \cdot C \quad \cdots(1) \\
& \Longleftrightarrow\left(\mu \circ \mu^{\prime}\right)_{*}(\tilde{g} \circ \tilde{h})^{*} H \cdot C \leq \mu_{*} \tilde{f}^{*} \nu_{*} \tilde{g}^{*} H \cdot C \\
& \Longleftrightarrow \mu_{*} \mu_{*}^{\prime} \tilde{h}^{*} \tilde{g}^{*} H \cdot C \leq \mu_{*} \tilde{f}^{*} \nu_{*} \tilde{g}^{*} H \cdot C .
\end{aligned}
$$

Here $\mu_{*} \tilde{f}^{*} \nu_{*} \tilde{g}^{*} H=\mu_{*} \mu_{*}^{\prime} \mu^{*} \tilde{f}^{*} \nu_{*} \tilde{g}^{*} H=\mu_{*} \mu_{*}^{\prime} \tilde{h}^{*} \nu^{*} \nu_{*} \tilde{g}^{*} H$. Set

$$
E=\nu^{*} \nu_{*} \tilde{g}^{*} H-\tilde{g}^{*} H
$$

then (1) is equivalent to the inequality

$$
\mu_{*} \mu_{*}^{\prime} \tilde{h}^{*} E \cdot C \geq 0 .
$$

By negativity lemma (cf. [KoMo, Lemma 3.39]), $E$ is an effective and $\nu$-exceptional divisor. Take a curve $C^{\prime}$ on $X^{\prime}$ such that $\mu\left(C^{\prime}\right)=C$ and a curve $C^{\prime \prime}$ on $X^{\prime \prime}$ such that $\mu^{\prime}\left(C^{\prime \prime}\right)=C^{\prime}$.

$\nu\left(\tilde{h}\left(C^{\prime \prime}\right)\right)=\tilde{f}\left(\mu^{\prime}\left(C^{\prime \prime}\right)\right)=\tilde{f}\left(C^{\prime}\right)=f(C) \not \subset I_{g}$, so $\tilde{h}\left(C^{\prime \prime}\right) \not \subset \operatorname{Exc}(\nu)$. In particular, $\tilde{h}\left(C^{\prime \prime}\right) \not \subset \operatorname{Supp} E$ and then $C^{\prime \prime} \not \subset \operatorname{Supp} \tilde{h}^{*} E$. Hence

$$
C=\mu\left(\mu^{\prime}\left(C^{\prime \prime}\right)\right) \not \subset \mu\left(\mu^{\prime}\left(\operatorname{Supp} \tilde{h}^{*} E\right)\right)=\operatorname{Supp} \mu_{*} \mu_{*}^{\prime} \tilde{h}^{*} E .
$$

This implies (1).

(ii) is obvious since $\left.f\right|_{C}: C \rightarrow f(C)$ and $\left.g\right|_{f(C)}: f(C) \rightarrow Y$ are morphisms.

The following lemma is a variant of Lemma 4.6.

Lemma 4.7. Let $f: X \rightarrow Y$ be a dominant rational map of smooth projective varieties and $g: C \rightarrow X$ a morphism from a curve $C$. Take a Cartier divisor $H$ on $Y$.

(i) If $g(C) \not \subset I_{f}$ and $H$ is nef, then

$$
\operatorname{deg}\left((f \circ g)^{*} H\right) \leq \operatorname{deg}\left(g^{*} f^{*} H\right) .
$$


(ii) If $g(C) \cap I_{f}=\varnothing$, then

$$
\operatorname{deg}\left((f \circ g)^{*} H\right)=\operatorname{deg}\left(g^{*} f^{*} H\right) .
$$

Proof. (i) Since a nef divisor is the limit of a sequence of ample divisors, we may assume that $H$ is ample.

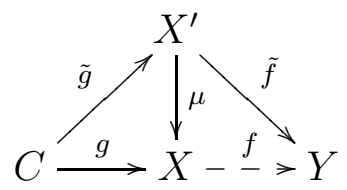

In the above diagram, $\tilde{f}: X^{\prime} \rightarrow Y$ is an elimination of indeterminacy of $f$ by blowing up smooth centers in $I_{f}$, and we can define the composition $\tilde{g}=\mu \circ g$ by the assumption that $g(C) \not \subset I_{f}$. Moreover it is a morphism.

We compute

$$
\begin{aligned}
\operatorname{deg}\left(g^{*} f^{*} H\right)-\operatorname{deg}\left((f \circ g)^{*} H\right) & =\operatorname{deg}\left(\tilde{g}^{*} \mu^{*} \mu_{*} \tilde{f}^{*} H-\tilde{g}^{*} \tilde{f}^{*} H\right) \\
& =\operatorname{deg}\left(\tilde{g}^{*} E\right),
\end{aligned}
$$

where we set $E=\mu^{*} \mu_{*} \tilde{f}^{*} H-\tilde{f}^{*} H$. By negativity lemma(cf. KoMo, Lemma 3.39]), $E$ is an effective and $\mu$-exceptional divisor on $X^{\prime}$. Moreover $\tilde{g}(C) \not \subset$ Supp $E$ since $\mu(\tilde{g}(C))=g(C) \not \subset I_{f}$ and $\mu(E) \subset I_{f}$. So $\operatorname{deg}\left(\tilde{g}^{*} E\right) \geq 0$.

(ii) is obvious since both $g: C \rightarrow g(C)$ and $\left.f\right|_{g(C)}: g(C) \rightarrow Y$ are morphisms.

Lemma 4.8. Let $X$ be a smooth projective variety with an ample Cartier divisor $H . \overline{\mathrm{Eff}}(X) \subset N^{1}(X)_{\mathbb{R}}$ denotes the pseudo-effective cone of $X$. Take a 1-cycle $Z \in N_{1}(X)_{\mathbb{R}}$. Then there is a constant $M>0$ such that

$$
(E \cdot Z) \leq M\left(E \cdot H^{\operatorname{dim} X-1}\right)
$$

holds for any $E \in \overline{\mathrm{Eff}}(X)$.

Proof. Note that $\left(E \cdot H^{\operatorname{dim} X-1}\right)>0$ for any $E \in \overline{\operatorname{Eff}}(X) \backslash\{0\}$ (see $[$ KaSi2, Lemma 20]). We define a function $f: \overline{\operatorname{Eff}}(X) \backslash\{0\} \rightarrow \mathbb{R}$ as

$$
f(E)=\frac{(E \cdot Z)}{\left(E \cdot H^{\operatorname{dim} X-1}\right)} .
$$

Take a norm $\|\cdot\|$ on $N^{1}(X)_{\mathbb{R}}$ and set $S=\{E \in \overline{\operatorname{Eff}}(X) \mid\|E\|=1\}$. Then we can take an upper bound $M>0$ of $\left.f\right|_{S}$ since $S$ is compact. But $f$ satisfies $f(c E)=f(E)$ for $E \in N^{1}(X)_{\mathbb{R}}$ and $c>0$, so $M$ is in fact an upper bound of $f$. This implies the claim. 
Lemma 4.9. Let $(X, f)$ be a dynamical system over $\overline{k(t)}$ with a model $\left(X_{C} \stackrel{\pi}{\rightarrow} C, f_{C}\right)$ over a curve $C$ or $k$. Then $\delta_{f}=\delta_{f_{C}}$.

Proof. We define the $k$-th dynamical degree and the $k$-th relative dynamical degree:

$$
\begin{gathered}
\lambda_{k}\left(f_{C}\right)=\lim _{m \rightarrow \infty}\left(\left(f_{C}^{m}\right)^{*} H_{C}^{k} \cdot H_{C}^{n+1-k}\right)^{1 / m}, \\
\lambda_{k}\left(f_{C} \mid \pi\right)=\lim _{m \rightarrow \infty}\left(\left(f_{C}^{m}\right)^{*} H_{C}^{k} \cdot H_{C}^{n-k} \cdot F\right)^{1 / m} .
\end{gathered}
$$

Note that $\lambda_{1}\left(f_{C}\right)=\delta_{f_{C}}$.

Set $n=\operatorname{dim} X$. Take an ample divisor $H_{C}$ on $X_{C}$ and a general fiber $F$ of $\pi$. Fix an integer $m>0$. Take an elimination of indeterminacy of $f_{C}^{m}$ :

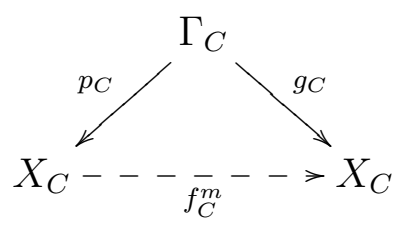

Pulling it back along $\overline{k(t)} \rightarrow C$, we get the following diagram:

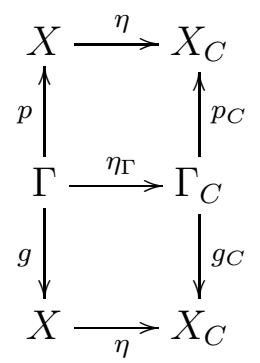

Set $H=\eta^{*} H_{C}$. We can show that $g^{*} \eta^{*}=\eta_{\Gamma}^{*} g_{C}^{*}$ and $p_{*} \eta_{\Gamma}^{*}=\eta^{*} p_{C *}$. So we have

$$
\left(f^{m}\right)^{*} H=p_{*} g^{*} \eta^{*} H_{C}=p_{*} \eta_{\Gamma}^{*} g_{C}^{*} H_{C}=\eta^{*} p_{C *} g_{C}^{*} H_{C}=\eta^{*}\left(f_{C}^{m}\right)^{*} H_{C} .
$$

So $\left(\left(f^{m}\right)^{*} H \cdot H^{n-1}\right)=\left(\eta^{*}\left(f_{C}^{m}\right)^{*} H_{C} \cdot\left(\eta^{*} H_{C}\right)^{n-1}\right)$. Hence $\left(\left(f^{m}\right)^{*} H \cdot H^{n-1}\right)$ is equal to the coefficient of the monomial $t_{1} \cdots t_{n}$ for the numerical polynomial

$$
\chi\left(X, t_{1} \eta^{*}\left(f_{C}^{m}\right)^{*} H_{C}+t_{2} \eta^{*} H_{C}+\cdots+t_{n} \eta^{*} H_{C}\right) .
$$

For any Cartier divisor $D$ on $X_{C}$ and a general fiber $F$ of $\pi$, the equality $\chi\left(X, \eta^{*} D\right)=\chi\left(F,\left.D\right|_{F}\right)$ holds. So

$$
\begin{aligned}
& \chi\left(X, t_{1} \eta^{*}\left(f_{C}^{m}\right)^{*} H_{C}+t_{2} \eta^{*} H_{C}+\cdots+t_{n} \eta^{*} H_{C}\right) \\
= & \chi\left(F,\left.t_{1}\left(f_{C}^{m}\right)^{*} H_{C}\right|_{F}+\left.t_{2} H_{C}\right|_{F}+\cdots+\left.t_{n} H_{C}\right|_{F}\right) .
\end{aligned}
$$

Hence we have $\left(\eta^{*}\left(f_{C}^{m}\right)^{*} H_{C} \cdot\left(\eta^{*} H_{C}\right)^{n-1}\right)=\left(\left.\left(f_{C}^{m}\right)^{*} H_{C}\right|_{F} \cdot\left(\left.H_{C}\right|_{F}\right)^{n-1}\right)=$ $\left(\left(f_{C}^{m}\right)^{*} H_{C} \cdot H_{C}^{n-1} \cdot F\right)$, and so $\lambda_{1}(f)=\lambda_{1}\left(f_{C} \mid \pi\right)$. 
On the other hand, by [Tru, Theorem 1.4],

$$
\begin{aligned}
\lambda_{1}\left(f_{C}\right) & =\max \left\{\lambda_{1}\left(f_{C} \mid \pi\right) \lambda_{0}\left(\operatorname{id}_{C}\right), \lambda_{0}\left(f_{C} \mid \pi\right) \lambda_{1}\left(\operatorname{id}_{C}\right)\right\} \\
& =\max \left\{\lambda_{1}\left(f_{C} \mid \pi\right), 1\right\} \\
& =\lambda_{1}\left(f_{C} \mid \pi\right) .
\end{aligned}
$$

Note that $\lambda_{q}\left(\operatorname{id}_{C}\right)=1$ for all $q$ and $\lambda_{0}\left(f_{C} \mid \pi\right)=1$ by definition. So

$$
\delta_{f}=\lambda_{1}(f)=\lambda_{1}\left(f_{C} \mid \pi\right)=\lambda_{1}\left(f_{C}\right)=\delta_{f_{C}} .
$$

Proof of Theorem 4.3. Take $P \in X_{f}$. Put $n=\operatorname{dim} X$. By Lemma 4.5, we can take a model $\left(X_{C} \stackrel{\pi}{\rightarrow} C, f_{C}\right)$ over a curve $C$. We may assume that $P$ corresponds to a section $\sigma: C \rightarrow X_{C}$ of $\pi$.

Take an ample Cartier divisor $H_{C}$ on $X_{C}$ and set $H=(X \rightarrow$ $\left.X_{C}\right)^{*} H_{C}$. By Lemma 4.9,

$$
\delta_{f}=\delta_{f_{C}}=\lim _{m \rightarrow \infty}\left(\left(f_{C}^{m}\right)^{*} H_{C} \cdot H_{C}^{n}\right)^{1 / m} .
$$

On the other hand, by Proposition 2.14,

$$
\begin{aligned}
\bar{\alpha}_{f}(P) & =\limsup _{m \rightarrow \infty} \tilde{h}_{H}^{+}\left(f^{m}(P)\right)^{1 / m} \\
& =\limsup _{m \rightarrow \infty} \operatorname{deg}^{+}\left(\left(f_{C}^{m} \circ \sigma\right)^{*} H_{C}\right)^{1 / m} .
\end{aligned}
$$

Note that $\operatorname{Im}(\sigma) \not \subset I_{f_{C}^{m}}$ since $P \notin I_{f^{m}}$. By Lemma 4.7 (i),

$$
\operatorname{deg}\left(\left(f_{C}^{m} \circ \sigma\right)^{*} H_{C}\right) \leq \operatorname{deg}\left(\sigma^{*}\left(f_{C}^{m}\right)^{*} H_{C}\right)=\left(\left(f_{C}^{m}\right)^{*} H_{C} \cdot \sigma_{*} C\right) .
$$

It is obvious that $\left(f_{C}^{m}\right)^{*} H_{C} \in \overline{\operatorname{Eff}}\left(X_{C}\right)$ for every $m$. So, by Lemma 4.8, there is a constant $M>0$ such that the inequality

$$
\left(\left(f_{C}^{m}\right)^{*} H_{C} \cdot \sigma_{*} C\right) \leq M\left(\left(f_{C}^{m}\right)^{*} H_{C} \cdot H_{C}^{n}\right)
$$

holds for every $m$. Therefore we have

$$
\begin{aligned}
\bar{\alpha}_{f}(P) & \leq \limsup _{m \rightarrow \infty}\left(\left(f_{C}^{m}\right)^{*} H_{C} \cdot \sigma_{*} C\right)^{1 / m} \\
& \leq \limsup _{m \rightarrow \infty}\left(M\left(\left(f_{C}^{m}\right)^{*} H_{C} \cdot H_{C}^{n}\right)\right)^{1 / m} \\
& =\limsup _{m \rightarrow \infty}\left(\left(f_{C}^{m}\right)^{*} H_{C} \cdot H_{C}^{n}\right)^{1 / m} \\
& =\delta_{f_{C}} \\
& =\delta_{f} .
\end{aligned}
$$




\section{A SUFFICIENT CONDITION}

Let $(X, f)$ be a dynamical system over $\overline{k(t)}$. In this section, we give a sufficient condition of a rational point $P \in X_{f}$ whose arithmetic degree attains the dynamical degree.

Theorem 5.1. Let $(X, f)$ be a dynamical system over $\overline{k(t)}$ and $\left(X_{C} \stackrel{\pi}{\rightarrow}\right.$ $\left.C, f_{C}\right)$ a model over a curve $C$. Take a rational point $P \in X_{f}$ corresponding to a section $\sigma: C \rightarrow X_{C}$ of $\pi$. Assume that

- $\sigma(C) \cap I_{f_{C}^{m}}=\varnothing$ for every $m \geq 1$ and

- $(E \cdot \sigma(C))>0$ for any $E \in \overline{\mathrm{Eff}}(X) \backslash\{0\}$.

Then $\alpha_{f}(P)$ exists and $\alpha_{f}(P)=\delta_{f}$.

We prepare the following lemma.

Lemma 5.2. Let $X$ be a smooth projective variety and $Z \subset X$ a 1cycle such that $(E \cdot Z)>0$ for any $E \in \overline{\mathrm{Eff}}(X) \backslash\{0\}$. We define a non-negative function $\|\cdot\|_{Z}: N^{1}(X)_{\mathbb{R}} \rightarrow \mathbb{R}$ as $\|v\|_{Z}=\inf \left\{\left(v_{1} \cdot Z\right)+\left(v_{2} \cdot Z\right) \mid v=v_{1}-v_{2}, v_{1}, v_{2}\right.$ are effective classes $\}$.

(i) $\|v\|_{Z}=(v \cdot Z)$ for any effective class $v \in N^{1}(X)_{\mathbb{R}}$.

(ii) $\|\cdot\|_{Z}$ is a norm on $N^{1}(X)_{\mathbb{R}}$.

Proof. (i) For effective classes $v_{1}, v_{2}$ such that $v=v_{1}-v_{2}$, we have $(v \cdot Z)=\left(v_{1} \cdot Z\right)-\left(v_{2} \cdot Z\right) \leq\left(v_{1} \cdot Z\right)+\left(v_{2} \cdot Z\right)$. So $\|v\|_{Z}=(v \cdot Z)$.

(ii) It is easy to see that

- $\|c v\|_{Z}=|c| \cdot\|v\|_{Z}$ for any $c \in \mathbb{R}$ and $v \in N^{1}(X)_{\mathbb{R}}$ and

- $\|v+w\|_{Z} \leq\|v\|_{Z}+\|w\|_{Z}$ for any $v, w \in N^{1}(X)_{\mathbb{R}}$.

Take $v \in N^{1}(X)_{\mathbb{R}}$ and assume that $\|v\|_{Z}=0$. Then we have

$$
\left\{v_{n}^{+}\right\}_{n},\left\{v_{n}^{-}\right\}_{n} \subset N^{1}(X)_{\mathbb{R}}
$$

such that $v=v_{n}^{+}-v_{n}^{-}$for every $n$ and

$$
\lim _{n \rightarrow \infty}\left(\left(v_{n}^{+} \cdot Z\right)+\left(v_{n}^{-} \cdot Z\right)\right)=0 .
$$

So $\lim _{n \rightarrow \infty}\left(v_{n}^{ \pm} \cdot Z\right)=0$. Since $(w \cdot Z)>0$ for any $w \in \overline{\operatorname{Eff}}(X) \backslash\{0\}$, it follows that $\lim _{n \rightarrow \infty} v_{n}^{ \pm}=0$. Therefore $v=\lim _{n \rightarrow \infty}\left(v_{n}^{+}-v_{n}^{-}\right)=0$. So $\|\cdot\|_{Z}$ satisfies the conditions of norm.

Proof of Theorem 5.1. Set $n=\operatorname{dim} X$. We have

$$
\begin{aligned}
\delta_{f} & =\delta_{f_{C}} \quad(\text { by Lemma 4.9) } \\
& =\lim _{m \rightarrow \infty}\left(\left(f_{C}^{m}\right)^{*} H_{C} \cdot H_{C}^{n}\right)^{1 / m} \\
& \left.=\lim _{m \rightarrow \infty}\left\|\left(f_{C}^{m}\right)^{*} H_{C}\right\|_{H_{C}^{n}}^{1 / m} . \quad \text { (by Lemma } 5.2(\mathrm{i})\right)
\end{aligned}
$$


Note that $\|\cdot\|_{H_{C}^{n}}$ is a norm since $\left(E \cdot H_{C}^{n}\right)>0$ for every $E \in \overline{\operatorname{Eff}}(X) \backslash\{0\}$ (cf. [KaSi2, Lemma 20]). We obtain

$$
\begin{aligned}
\delta_{f} & =\lim _{m \rightarrow \infty}\left\|\left(f_{C}^{m}\right)^{*} H_{C}\right\|_{\sigma(C)}^{1 / m} \quad\left(\text { since }\|\cdot\|_{H_{C}^{n}} \text { is equivalent to }\|\cdot\|_{\sigma(C)}\right) \\
& =\lim _{m \rightarrow \infty}\left(\left(f_{C}^{m}\right)^{*} H_{C} \cdot \sigma(C)\right)^{1 / m} \quad(\text { by Lemma 5.2 }(\mathrm{i})) \\
& =\lim _{m \rightarrow \infty} \operatorname{deg}^{+}\left(\sigma^{*}\left(f_{C}^{m}\right)^{*} H_{C}\right)^{1 / m} \\
& =\lim _{m \rightarrow \infty} \operatorname{deg}^{+}\left(\left(f_{C}^{m} \circ \sigma\right)^{*} H_{C}\right)^{1 / m} \quad \text { (by Lemma 4.7 (ii)) } \\
& =\lim _{m \rightarrow \infty} \tilde{h}_{H}^{+}\left(f^{m}(P)\right)^{1 / m} \quad(\text { by Proposition 2.14) } \\
& =\alpha_{f}(P) .
\end{aligned}
$$

\section{Arithmetic Degrees For Projective spaCeS}

In this section, we study arithmetic degrees for dynamical systems on projective spaces.

At first, we give some sufficient conditions for a rational point at which the arithmetic degree attains the dynamical degree.

Lemma 6.1. Let $C$ be a curve. Set $X=\mathbb{P}_{k}^{n} \times C$. Take an pseudoeffective Cartier divisor $E$ on $X$ and a general fiber $F$ of $\pi=\operatorname{pr}_{C}$. Then $\mathcal{O}_{X}(E) \equiv \mathcal{O}_{X}(d) \otimes \mathcal{O}_{X}(e F)$ for some $d, e \in \mathbb{Z}_{\geq 0}$.

Proof. It is sufficient to prove the claim for effective divisors, so we may assume that $E$ is effective. Since $\operatorname{Pic}(X)$ is generated by $\mathcal{O}_{X}(1)$ and $\pi^{*} \operatorname{Pic}(C)$, there are an integer $d$ and a divisor $D_{C}$ on $C$ such that $\mathcal{O}_{X}(E) \cong \mathcal{O}_{X}(d) \otimes \pi^{*} \mathcal{O}_{C}\left(D_{C}\right)$. Set $e=\operatorname{deg} D_{C}$. Then $\mathcal{O}_{X}(E) \equiv$ $\mathcal{O}_{X}(d) \otimes \mathcal{O}_{X}(e F)$.

Since $\left.E\right|_{F}$ is effective and $\mathcal{O}_{F}\left(\left.E\right|_{F}\right) \cong \mathcal{O}_{\mathbb{P}_{\mathbb{C}}^{n}}(d), d \geq 0$. By projection formula,

$\pi_{*}\left(\mathcal{O}_{X}(d) \otimes \pi^{*} \mathcal{O}_{C}\left(D_{C}\right)\right) \cong \pi_{*}\left(\mathcal{O}_{X}(d)\right) \otimes \mathcal{O}_{C}\left(D_{C}\right) \cong S^{d}\left(\mathcal{O}_{C}^{\oplus n+1}\right) \otimes \mathcal{O}_{C}\left(D_{C}\right)$. Since $H^{0}\left(C, S^{d}\left(\mathcal{O}_{C}^{\oplus n+1}\right) \otimes \mathcal{O}_{C}\left(D_{C}\right)\right)=H^{0}\left(X, \mathcal{O}_{X}(d) \otimes \pi^{*} \mathcal{O}_{C}\left(D_{C}\right)\right) \neq 0$, $D_{C}$ is effective. So $e=\operatorname{deg} D_{C} \geq 0$.

Theorem 6.2. Let $\left(X=\mathbb{P} \frac{n}{k(t)}, f\right)$ be a dynamical system over $\overline{k(t)}$ and $\left(X_{C}=\mathbb{P}_{k}^{n} \times C \stackrel{\mathrm{pr}_{C}}{\rightarrow} C, f_{C}\right)$ a model of $(X, f)$ over a curve $C$. Take a morphism $g: C \rightarrow \mathbb{P}_{k}^{n}$ corresponding to a rational point $P_{g} \in X_{f}$ and set $\sigma_{g}=\left(g, \operatorname{id}_{C}\right): C \rightarrow X_{C}$.

(i) Assume that $g$ is non-constant and $\operatorname{Im}\left(\sigma_{g}\right) \cap I_{f_{C}^{m}}=\varnothing$ for every $m \geq 1$. Then $\alpha_{f}\left(P_{g}\right)$ exists and $\alpha_{f}\left(P_{g}\right)=\delta_{f}$. 
(ii) Assume the following conditions.

(*) For every $m \geq 0, \operatorname{pr}_{\mathbb{P}_{k}^{n}} \circ f_{C}^{m} \circ \sigma_{g}$ is non-constant.

(**) There is a sequence of positive integers $m_{1}<m_{2}<\ldots$ such that $\operatorname{Im}\left(f_{C}^{m_{k}} \circ \sigma_{g}\right) \cap I_{f_{C}^{m_{k+1}-m_{k}}}=\varnothing$ for every $k \geq 1$ and $\lim _{k \rightarrow \infty}\left(m_{k} / m_{k+1}\right)=0$.

$(* * *)$ The limit $\alpha_{f}\left(P_{g}\right)$ exists.

Then the equality $\alpha_{f}\left(P_{g}\right)=\delta_{f}$ holds.

Proof. (i) Let $F$ be a general fiber of $\operatorname{pr}_{C}$. Then

$$
\overline{\operatorname{Eff}}\left(X_{C}\right)=\mathbb{R}_{\geq 0} \mathcal{O}_{X}(F)+\mathbb{R}_{\geq 0} \mathcal{O}_{X}(1)
$$

by Lemma 6.1. It is obvious that $\sigma_{g}$ satisfies the assuption of Theorem 5.1. So (i) follows from Theorem 5.1.

(ii) For $m \geq 1$, set $\left(f_{C}^{m}\right)^{*} \mathcal{O}_{X_{C}}(1) \equiv \mathcal{O}_{X_{C}}\left(d_{m}\right) \otimes \mathcal{O}_{X_{C}}\left(e_{m} F\right)$. Then $d_{m}, e_{m} \geq 0$ by Lemma 6.1. It is clear that $\left(f^{m}\right)^{*} \mathcal{O}_{X}(1) \equiv \mathcal{O}_{X}\left(d_{m}\right)$, and so

$$
\delta_{f}=\lim _{m \rightarrow \infty}\left(\left(f^{m}\right)^{*} \mathcal{O}_{X}(1) \cdot \mathcal{O}_{X}(1)^{n-1}\right)^{1 / m}=\lim _{m \rightarrow \infty} d_{m}^{1 / m} .
$$

Set $b_{m}=\operatorname{deg}\left(\left(f_{C}^{m} \circ \sigma_{g}\right)^{*} \mathcal{O}_{X_{C}}(1)\right)$. By $(*), b_{m} \geq 1$ for every $m$. So we have

$$
\alpha_{f}\left(P_{g}\right)=\lim _{m \rightarrow \infty} \max \left\{b_{m}, 1\right\}^{1 / m}=\lim _{m \rightarrow \infty} b_{m}^{1 / m} .
$$

For $k \geq 1$, set $l_{k}=m_{k+1}-m_{k}$. We compute

$$
\begin{aligned}
b_{m_{k+1}} & =\operatorname{deg}\left(\left(f_{C}^{m_{k+1}} \circ \sigma_{g}\right)^{*} \mathcal{O}_{X_{C}}(1)\right) \\
& \left.=\operatorname{deg}\left(f_{C}^{l_{k}} \circ f_{C}^{m_{k}} \circ \sigma_{g}\right)^{*} \mathcal{O}_{X_{C}}(1)\right) \\
& \left.=\operatorname{deg}\left(f_{C}^{m_{k}} \circ \sigma_{g}\right)^{*}\left(f_{C}^{l_{k}}\right)^{*} \mathcal{O}_{X_{C}}(1)\right) \quad(\text { by }(* *) \text { and Lemma 4.7 (ii) }) \\
& =\operatorname{deg}\left(\left(f_{C}^{m_{k}} \circ \sigma_{g}\right)^{*} \mathcal{O}_{X_{C}}\left(d_{l_{k}}\right) \otimes \mathcal{O}_{X_{C}}\left(e_{l_{k}} F\right)\right) \\
& \geq \operatorname{deg}\left(\left(f_{C}^{m_{k}} \circ \sigma_{g}\right)^{*} \mathcal{O}_{X_{C}}\left(d_{l_{k}}\right)\right) \\
& =d_{l_{k}} b_{m_{k}} \\
& \geq d_{l_{k}} .
\end{aligned}
$$

Note that $\lim _{k \rightarrow \infty} l_{k}=\infty$ by the assumption that $\lim _{k \rightarrow \infty}\left(m_{k} / m_{k+1}\right)=$ 0 . Hence

$$
\begin{aligned}
\alpha_{f}\left(P_{g}\right) & =\lim _{m \rightarrow \infty}\left(b_{m}\right)^{\frac{1}{m}} \quad \text { (by Proposition 2.14) } \\
& =\lim _{k \rightarrow \infty}\left(b_{m_{k+1}}\right)^{\frac{1}{m_{k+1}}} \\
& \geq \lim _{k \rightarrow \infty}\left(d_{l_{k}}\right)^{\frac{1}{l_{k}} \cdot\left(1-\frac{m_{k}}{m_{k+1}}\right)} \\
& =\delta_{f} .
\end{aligned}
$$

Combining with Theorem 4.1, it follows that $\alpha_{f}\left(P_{g}\right)=\delta_{f}$. 
Next, we show that a sufficiently general morphism $g: C \rightarrow \mathbb{P}_{k}^{n}$ of a given sufficiently large degree corresponds to a rational point whose arithmetic degree attains the dynamical degree.

Definition 6.3. Let $C$ be a curve of genus $g(C)$ over $k$ and $d, n$ positive integers. $\operatorname{Mor}_{d}\left(C, \mathbb{P}_{k}^{n}\right)$ denotes the set of morphisms $g: C \rightarrow \mathbb{P}_{k}^{n}$ such that $\operatorname{deg}\left(g^{*} \mathcal{O}(1)\right)=d$.

$\operatorname{Mor}_{d}\left(C, \mathbb{P}_{k}^{n}\right)$ has a structure of $k$-variety with the evaluation $e$ : $\operatorname{Mor}_{d}\left(C, \mathbb{P}_{k}^{n}\right) \times C \rightarrow \mathbb{P}_{k}^{n}$ which maps $(g, p)$ to $g(p)$. Moreover, if $\operatorname{Mor}_{d}\left(C, \mathbb{P}_{k}^{n}\right)$ is non-empty, we have

$$
\operatorname{dim} \operatorname{Mor}_{d}\left(C, \mathbb{P}_{k}^{n}\right) \geq(n+1) d+n(1-g(C))
$$

(cf. [KoMo, 1.1]).

Theorem 6.4. Let $\left(X=\mathbb{P}_{\overline{k(t)}}^{n}, f\right)$ be a dynamical system over $\overline{k(t)}$ and $\left(X_{C}=\mathbb{P}_{k} \times C \stackrel{\mathrm{pr}_{C}}{\rightarrow} C, f_{C}\right)$ a model of $(X, f)$ over a curve $C$ of genus $g(C)$. Take a positive integer $d$ satisfying $d>\frac{n(g(C)-1)}{n+1} . P_{g} \in$ $X(\overline{k(t)})$ denotes the rational point corresponding to $g \in \operatorname{Mor}\left(C, \mathbb{P}_{k}^{n}\right)$. Then $\alpha_{f}\left(P_{g}\right)$ exists and $\alpha_{f}\left(P_{g}\right)=\delta_{f}$ for a sufficiently general $g \in$ $\operatorname{Mor}_{d}\left(C, \mathbb{P}_{k}^{n}\right)$.

Proof. Let $M \subset \operatorname{Mor}_{d}\left(C, \mathbb{P}_{k}^{n}\right)$ be an irreducible component of maximal dimension. Then $\operatorname{dim} M>0$ by assumption. Set $\Phi=\left(e, \operatorname{id}_{C}\right): M \times$ $C \rightarrow X_{C}$, where $e$ is the evaluation. For any $g \in M$ and $\rho \in \operatorname{Aut}\left(\mathbb{P}_{k}^{n}\right)$, we have $\operatorname{deg}\left(g^{*} \rho^{*} \mathcal{O}_{\mathbb{P}^{n}}(1)\right)=\operatorname{deg}\left(g^{*} \mathcal{O}_{\mathbb{P}^{n}}(1)\right)=d$, so $\operatorname{Aut}\left(\mathbb{P}_{k}^{n}\right)$ acts on $M$. Fix $g_{0} \in M$. For any $(x, p) \in X_{C}$, we can take $\rho \in \operatorname{Aut}\left(\mathbb{P}_{k}^{n}\right)$ such that $\rho\left(g_{0}(p)\right)=x$. Then $\Phi\left(\rho \circ g_{0}, p\right)=\left(\left(\rho \circ g_{0}\right)(p), p\right)=(x, p)$. So it follows that $\Phi$ is surjective.

For every $m \geq 1$, we compute

$$
\begin{aligned}
\operatorname{dim} \Phi^{-1}\left(I_{f_{C}^{m}}\right) & \leq\left(\operatorname{dim}(M \times C)-\operatorname{dim} X_{C}\right)+\operatorname{dim} I_{f_{C}^{m}} \\
& \leq\left(\operatorname{dim} M+1-\operatorname{dim} X_{C}\right)+\operatorname{dim} X_{C}-2 \\
& =\operatorname{dim} M-1 .
\end{aligned}
$$

Hence $\operatorname{pr}_{M}\left(\Phi^{-1}\left(I_{f_{C}^{m}}\right)\right) \subset M$ is a proper subset of $M$ for every $m \geq 1$.

For $g \in M, \sigma_{g}=\left(g, \mathrm{id}_{C}\right): C \rightarrow X_{C}$ denotes the corresponding section of $\operatorname{pr}_{C}$. For $g \in M$, we have

$$
\begin{aligned}
\sigma_{g}(C) \cap I_{f_{C}^{m}}=\varnothing & \Longleftrightarrow \Phi(\{g\} \times C) \cap I_{f_{C}^{m}}=\varnothing \\
& \Longleftrightarrow\{g\} \times C \cap \Phi^{-1}\left(I_{f_{C}^{m}}\right)=\varnothing \\
& \Longleftrightarrow g \notin \operatorname{pr}_{M}\left(\Phi^{-1}\left(I_{f_{C}^{m}}\right)\right) .
\end{aligned}
$$


Set $\left(f_{C}^{m}\right)^{*} \mathcal{O}_{X_{C}}(1) \equiv \mathcal{O}_{X_{C}}\left(d_{m}\right) \otimes \mathcal{O}_{X_{C}}\left(e_{m} F\right)$, where $F$ is a general fiber of $\operatorname{pr}_{C}$. Then $d_{m}, e_{m} \geq 0$ by Lemma6.1. Take $g \in M \backslash \bigcup_{m \geq 1} \operatorname{pr}_{M}\left(\Phi^{-1}\left(I_{f_{C}^{m}}\right)\right)$.

We compute

$$
\begin{aligned}
\underline{\alpha}_{f}\left(P_{g}\right) & =\liminf _{m \rightarrow \infty} \operatorname{deg}\left(\left(f_{C}^{m} \circ \sigma_{g}\right)^{*} \mathcal{O}_{X_{C}}(1)\right)_{+}^{1 / m} \quad(\text { by Proposition 2.14) } \\
& =\liminf _{m \rightarrow \infty} \operatorname{deg}\left(\sigma_{g}^{*}\left(f_{C}^{m}\right)^{*} \mathcal{O}_{X_{C}}(1)\right)_{+}^{1 / m} \quad(\text { by Lemma 4.7 (ii) }) \\
& =\liminf _{m \rightarrow \infty}\left(\mathcal{O}_{X_{C}}\left(d_{m}\right) \otimes \mathcal{O}_{X_{C}}\left(e_{m} F\right) \cdot \sigma_{g *} C\right)_{+}^{1 / m} \\
& \geq \liminf _{m \rightarrow \infty}\left(\mathcal{O}_{X_{C}}\left(d_{m}\right) \cdot \sigma_{g *} C\right)_{+}^{1 / m} \\
& =\liminf _{m \rightarrow \infty}\left(d_{m}\left(\mathcal{O}_{X_{C}}(1) \cdot \sigma_{g *} C\right)\right)_{+}^{1 / m} \\
& =\liminf _{m \rightarrow \infty} d_{m}^{1 / m} \\
& =\delta_{f} .
\end{aligned}
$$

Note that $\left(\mathcal{O}_{X_{C}}(1) \cdot \sigma_{g *} C\right)=\left(\mathcal{O}_{\mathbb{P}_{k}^{n}}(1) \cdot g_{*} C\right)>0$ since $d=\operatorname{deg}(g)>$ 0 . Combining with Theorem 4.3, it follows that $\alpha_{f}\left(P_{g}\right)$ exists and $\alpha_{f}\left(P_{g}\right)=\delta_{f}$.

\section{Construction of orbits}

In this section, we consider a problem on the existence of the rational points at which the arithmetic degree attains the dynamical degree.

Over $\overline{\mathbb{Q}}$, the following problem is studied in some papers (cf. KKaSi1, Theorem 3] and [MSS, Theorem 1.7]).

Problem 7.1. Let $(X, f)$ be a dynamical system over $\overline{\mathbb{Q}}$. Is there a subset $S \subset X_{f}$ such that

- $\alpha_{f}(P)$ exists and $\alpha_{f}(P)=\delta_{f}$ for every $P \in S$,

- $O_{f}(P) \cap O_{f}(Q)=\varnothing$ if $P, Q \in S$ and $P \neq Q$, and

- $S$ is a Zariski dense subset of $X$.

or not?

We give an affirmative answer for any dynamical system over $\overline{k(t)}$, where $k$ is an uncountable algebraically closed field of characteristic 0 .

Theorem 7.2. Assume that $k$ is an uncountable algebraically closed field of characteristic 0 . Let $(X, f)$ be a dynamical system over $\overline{k(t)}$. Then there exists a subset $S \subset X_{f}$ such that

- $\alpha_{f}(P)$ exists and $\alpha_{f}(P)=\delta_{f}$ for every $P \in S$,

- $O_{f}(P) \cap O_{f}(Q)=\varnothing$ if $P, Q \in S$ and $P \neq Q$, and

- $S$ is a Zariski dense subset of $X$. 
Lemma 7.3. Let $k$ be an uncountable algebraically closed field and $X$ an algebraic scheme of positive dimension over $k$. Let $Z_{1}, Z_{2}, \ldots \subset X$ be proper closed subsets of $X$. Then $\bigcup_{i} Z_{i} \neq X$ and there exists a countable set $M$ of $k$-valued points of $X \backslash \bigcup_{i} Z_{i}$ such that $M$ is Zariski dense in $X$.

Proof. Replacing $X$ by an affine open subset, we may assume that $X$ is affine. By Noether's normalization lemma, there is a finite cover $\phi: X \rightarrow \mathbb{A}_{k}^{n}$. Replacing $X$ and $Z_{1}, Z_{2}, \ldots$ by $\mathbb{A}_{k}^{n}$ and $\phi\left(Z_{1}\right), \phi\left(Z_{2}\right), \ldots$, we may assume that $X=\mathbb{A}_{k}^{n}$.

We prove the claim by induction on $n$. Assume that $n=1$. Then $Z_{i}(k)$ is a finite set for every $i$ and $\mathbb{A}_{k}^{1}(k)=k$ is uncountable, so $\bigcup_{i} Z_{i} \neq$ $\mathbb{A}_{k}^{1}$. We take an infinite subset $M$ of $\mathbb{A}_{k}^{1}(k) \backslash \bigcup_{i} Z_{i}(k)$. Then $M$ is a Zariski dense subset of $\mathbb{A}_{k}^{1}$.

Assume that the claim holds for $\mathbb{A}_{k}^{1}, \mathbb{A}_{k}^{2}, \ldots, \mathbb{A}_{k}^{n-1}$. Define $p: \mathbb{A}_{k}^{n} \rightarrow$ $\mathbb{A}_{k}^{n-1}$ and $q: \mathbb{A}_{k}^{n} \rightarrow \mathbb{A}_{k}^{1}$ as $p\left(x_{1}, \ldots, x_{n}\right)=\left(x_{1}, \ldots, x_{n-1}\right)$ and $q\left(x_{1}, \ldots, x_{n}\right)=$ $x_{n}$. Let $\left\{Z_{j}^{\prime}\right\}_{j}$ (resp. $\left.\left\{Z_{k}^{\prime \prime}\right\}_{k}\right)$ be the members of $\left\{Z_{i}\right\}_{i}$ such that $p\left(Z_{j}^{\prime}\right) \neq$ $\mathbb{A}_{k}^{n-1}$ (resp. $\left.p\left(Z_{k}^{\prime \prime}\right)=\mathbb{A}_{k}^{n-1}\right)$. Let $W_{k} \subset \mathbb{A}_{k}^{n-1}$ be the set of points $w \in \mathbb{A}_{k}^{n-1}$ such that the fiber $\left(\left.p\right|_{Z_{k}^{\prime \prime}}\right)^{-1}(w)=p^{-1}(w) \cap Z_{k}^{\prime \prime}$ of $\left.p\right|_{Z_{k}^{\prime \prime}}$ over $w$ has positive dimension. Then $W_{k}$ is a proper closed subset of $\mathbb{A}_{k}^{n-1}$. By induction hypothesis, $\bigcup_{j} \phi\left(Z_{j}^{\prime}\right) \cup \bigcup_{k} W_{k} \neq \mathbb{A}_{k}^{n-1}$ and we can take a countable subset $M^{\prime} \subset \mathbb{A}_{k}^{n-1}(k) \backslash\left(\bigcup_{j} \phi\left(Z_{j}^{\prime}\right)(k) \cup \bigcup_{k} W_{k}(k)\right)$ such that $M^{\prime}=\left\{a_{m}\right\}_{m=1}^{\infty}$ is Zariski dense in $\mathbb{A}_{k}^{n-1}$. For every $m$ and $k$, $p^{-1}\left(a_{m}\right) \cap Z_{k}^{\prime \prime} \neq \mathbb{A}_{k}^{1}$ since $a_{m} \notin W_{k}$. So $\bigcup_{m, k}\left(p^{-1}\left(a_{m}\right) \cap Z_{k}^{\prime \prime}\right) \not \subset \mathbb{A}_{k}^{1}$ and we can take a countable subset $M^{\prime \prime} \subset \mathbb{A}_{k}^{1}(k) \backslash \bigcup_{m, k}\left(p^{-1}\left(a_{m}\right)(k) \cap Z_{k}^{\prime \prime}(k)\right)$ such that $M^{\prime \prime}$ is Zariski dense in $\mathbb{A}_{k}^{1}$, by induction hypothesis. Set $M=M^{\prime} \times M^{\prime \prime} \subset \mathbb{A}_{k}^{n-1} \times \mathbb{A}_{k}^{1}$. Then it is clear that $M$ satisfies the claim.

Proof of Theorem 7.2. Take a model $\left(X_{C_{0}} \stackrel{\pi_{C_{0}}}{\rightarrow} C_{0}, f_{C_{0}}\right)$ of $(X, f)$ over a curve $C_{0}$. For any curve $C$ with a finite morphism $C \rightarrow C_{0},\left(X_{C} \stackrel{\pi_{C}}{\rightarrow}\right.$ $\left.C, f_{C}\right)$ denotes the pull-back of $\left(X_{C_{0}} \stackrel{\pi_{C_{0}}}{\rightarrow} C_{0}, f_{C_{0}}\right)$ by $C \rightarrow C_{0}$ and $\psi_{C}: X_{C} \rightarrow X_{C_{0}}$ denote the projection. For a section $\sigma: C \rightarrow X$ and a finite morphism $C^{\prime} \rightarrow C$ of curves, $(\sigma)_{C^{\prime}}: C^{\prime} \rightarrow X \times_{C} C^{\prime}$ denotes the pull-back of $\sigma$ by $C^{\prime} \rightarrow C$.

By Lemma 7.3, we can take a countable subset $M=\left\{a_{i}\right\}_{i=1}^{\infty} \subset X_{C_{0}}$ such that

- $M$ is Zariski dense in $X_{C_{0}}$ and

- $a_{i} \notin I_{f_{C_{0}}^{m}}$ for every $m \geq 1$ and $i \geq 1$.

We will construct rational points $P_{1}, P_{2}, \ldots \in X$ inductively. Let $C_{k} \rightarrow C_{k-1} \rightarrow \cdots \rightarrow C_{1} \rightarrow C_{0}$ be a sequence of finite morphisms of 
curves and $P_{i} \in X$ a rational point corresponding to a section $\sigma_{i}: C_{i} \rightarrow$ $X_{C_{i}}$ of $\pi_{C_{i}}$ for each $1 \leq i \leq k$. Assume that $P_{1}, \ldots, P_{k} \in X$ satisfy the following condition $(*)_{k}$ :

- $P_{i} \in X_{f}$ for $1 \leq i \leq k$,

- $\alpha_{f}\left(P_{i}\right)=\delta_{f}$ for $1 \leq i \leq k$,

- $O_{f}\left(P_{i}\right) \cap O_{f}\left(P_{j}\right)=\varnothing$ if $1 \leq i, j \leq k$ and $i \neq j$, and

- $a_{i} \in \operatorname{Im}\left(\psi_{C_{i}} \circ \sigma_{i}\right)$ for $1 \leq i \leq k$.

Set $n=\operatorname{dim} X$. Note that $X_{C_{k}}$ is smooth outside a finite union of fibers of $\pi_{C_{k}}$.

Let $p_{k}: X_{k} \rightarrow X_{C_{k}}$ be a resolution of $\left(X_{C_{k}}\right)_{\text {red }}$ whose exceptional locus is contained in a finite union of fibers of $\pi_{C_{k}}$. By blowing up a point in $\left(p_{k} \circ \psi_{C_{k}}\right)^{-1}\left(a_{k+1}\right)$, we may assume that $\left(p_{k} \circ \psi_{C_{k}}\right)^{-1}\left(a_{k+1}\right)$ has codimension 1 . We take a very ample divisor $H$ on $X_{k}$ and suitable members $H_{1}, \ldots, H_{n} \in|H|$, and set $C_{k+1}=H_{1} \cap \cdots \cap H_{n}$. Let $\iota: C_{k+1} \rightarrow X_{k}$ denote the inclusion. We can choose $H_{1}, \ldots, H_{n}$ as satisfying

(I) $C_{k+1}$ is a smooth and irreducible curve satisfying $\operatorname{Im}\left(\pi_{C_{k}} \circ p_{k} \circ\right.$ $\iota)=C_{k}$,

(II) $C_{k+1} \not \subset p_{k}^{-1}\left(f_{C_{k}}^{m}\right)^{-1}\left(I_{f_{C_{k}}}\right)$ for every $m \geq 0$,

(III) $C_{k+1} \cap I_{f_{k}^{m}}=\varnothing$ for every $m \geq 1$,

(IV) $C_{k+1} \not \subset\left(f_{k}^{m^{\prime}}\right)^{-1}\left(\operatorname{Im}\left(f_{k}^{m} \circ p_{k}^{-1} \circ\left(\sigma_{i}\right)_{C_{k}}\right)\right)$ for every $m, m^{\prime}$ and $1 \leq$ $i \leq k$, and

(V) $a_{k+1} \in \operatorname{Im}\left(\psi_{C_{k}} \circ p_{k} \circ \iota\right)$.

Set $\phi=\pi_{C_{k}} \circ p_{k} \circ \iota: C_{k+1} \rightarrow C_{k}$. Then we obtain the following diagram:

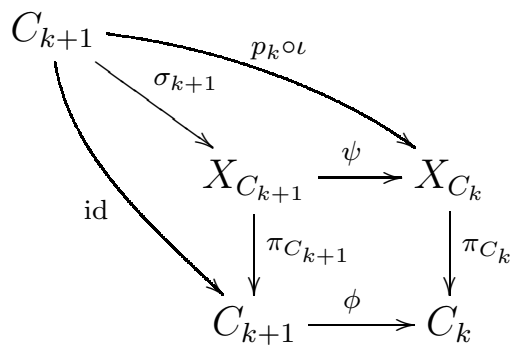

Here $X_{C_{k+1}}=X_{C_{k}} \times_{C_{k}} C_{k+1}$ and $\sigma_{k+1}$ is the unique morphism which makes the above diagram commutative. Let $P_{k+1} \in X$ be the rational point of $X$ corresponding to $\sigma_{k+1}$. By (II), $\operatorname{Im}\left(\sigma_{k+1}\right) \not \subset\left(f_{C_{k+1}}^{m}\right)^{-1}\left(I_{f_{C_{k+1}}}\right)$ for every $m \geq 0$. Hence $\operatorname{Im}\left(f_{C_{k+1}}^{m} \circ \sigma_{k+1}\right) \not \subset I_{f_{C_{k+1}}}$ and so $f^{m}\left(P_{k+1}\right) \notin I_{f}$ for every $m \geq 0$. Therefore $P_{k+1} \in X_{f}$.

Let $p_{k+1}: X_{k+1} \rightarrow X_{C_{k+1}}$ be a resolution of $\left(X_{C_{k+1}}\right)_{\text {red }}$ whose exceptional locus is over a finite union of fibers of $\pi_{C_{k+1}}$ and $\theta=p_{k}^{-1} \circ \psi \circ p_{k+1}$ 
becomes a morphism. Then we obtain the following diagram:

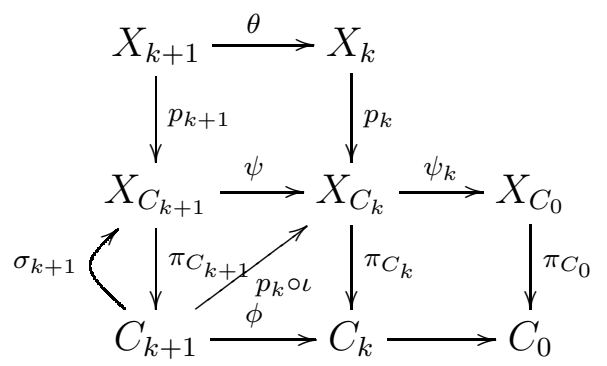

Set $f_{k}=p_{k}^{-1} \circ f_{C_{k}} \circ p_{k}, f_{k+1}=p_{k+1}^{-1} \circ f_{C_{k+1}} \circ p_{k+1}$ and $\sigma_{k+1}^{\prime}=p_{k+1}^{-1} \circ \sigma_{k+1}$. Fix a positive integer $m$. Then it follows that $p_{k} \circ \theta \circ f_{k+1}^{m} \circ \sigma_{k+1}^{\prime}=$ $p_{k} \circ f_{k}^{m} \circ \iota$. Since $p_{k}$ is birational, we have $\theta \circ f_{k+1}^{m} \circ \sigma_{k+1}^{\prime}=f_{k}^{m} \circ \iota$. Take an ample divisor $A$ on $X_{k+1}$ such that $A-\theta^{*} H$ is ample. We compute

$$
\begin{aligned}
\operatorname{deg}\left(f_{k+1}^{m} \circ \sigma_{k+1}^{\prime}\right)^{*} A & \geq \operatorname{deg}\left(f_{k+1}^{m} \circ \sigma_{k+1}^{\prime}\right)^{*} \theta^{*} H \\
& =\operatorname{deg}\left(\theta \circ f_{k+1}^{m} \circ \sigma_{k+1}^{\prime}\right)^{*} H \\
& =\operatorname{deg}\left(f_{k}^{m} \circ \iota\right)^{*} H .
\end{aligned}
$$

By (III) and Lemma 4.7 (ii), we have

$$
\operatorname{deg}\left(f_{k}^{m} \circ \iota\right)^{*} H=\operatorname{deg} \iota^{*}\left(f_{k}^{m}\right)^{*} H=\left(\left(f_{k}^{m}\right)^{*} H \cdot H^{n-1}\right) .
$$

Now $\left(X_{k+1} \stackrel{\pi_{C_{k+1}}{ }^{\circ p_{k+1}} \longrightarrow}{\longrightarrow} C_{k+1}, f_{k+1}\right)$ is a model of $(X, f)$ and $\sigma_{k+1}^{\prime}$ is a section of $\pi_{C_{k+1}} \circ p_{k+1}$ corresponding to $P_{k+1}$. Therefore

$$
\begin{aligned}
\underline{\alpha}_{f}\left(P_{k+1}\right) & =\liminf _{m \rightarrow \infty} \operatorname{deg}\left(\left(f_{k+1}^{m} \circ \sigma_{k+1}^{\prime}\right)^{*} A\right)^{1 / m} \quad \text { (by Proposition 2.14) } \\
& \geq \liminf _{m \rightarrow \infty}\left(\left(f_{k}^{m}\right)^{*} H \cdot H^{n-1}\right)^{1 / m} \\
& =\delta_{f_{k}}=\delta_{f} .
\end{aligned}
$$

So $\alpha_{f}\left(P_{k+1}\right)$ exists and $\alpha_{f}\left(P_{k+1}\right)=\delta_{f}$.

Fix $i \in\{0, \ldots, k\}$ and $m, m^{\prime} \geq 0$. By (IV), $\operatorname{Im}\left(f_{k}^{m^{\prime}} \circ \iota\right) \neq \operatorname{Im}\left(f_{k}^{m} \circ\right.$ $\left.p_{k}^{-1} \circ\left(\sigma_{i}\right)_{C_{k}}\right)=\operatorname{Im}\left(f_{k}^{m} \circ p_{k}^{-1} \circ\left(\sigma_{i}\right)_{C_{k}} \circ \phi\right)$. Since $p_{k}$ is birational and the images of both $f_{k}^{m^{\prime}} \circ \iota$ and $f_{k}^{m} \circ p_{k}^{-1} \circ\left(\sigma_{i}\right)_{C_{k}} \circ \phi$ intersects the isomorphic locus of $p_{k}$, we have

$$
\operatorname{Im}\left(p_{k} \circ f_{k}^{m^{\prime}} \circ \iota\right) \neq \operatorname{Im}\left(p_{k} \circ f_{k}^{m} \circ p_{k}^{-1} \circ\left(\sigma_{i}\right)_{C_{k}} \circ \phi\right) .
$$

On the other hand,

$$
p_{k} \circ f_{k}^{m^{\prime}} \circ \iota=\psi \circ f_{C_{k+1}}^{m^{\prime}} \circ \sigma_{k+1}
$$

and

$$
p_{k} \circ f_{k}^{m} \circ p_{k}^{-1} \circ\left(\sigma_{i}\right)_{C_{k}} \circ \phi=\psi \circ f_{C_{k+1}}^{m} \circ\left(\sigma_{i}\right)_{C_{k+1}} .
$$


So $\operatorname{Im}\left(\psi \circ f_{C_{k+1}}^{m^{\prime}} \circ \sigma_{k+1}\right) \neq \operatorname{Im}\left(\psi \circ f_{C_{k+1}}^{m} \circ\left(\sigma_{i}\right)_{C_{k+1}}\right)$, and so $f_{C_{k+1}}^{m^{\prime}} \circ$ $\sigma_{k+1} \neq f_{C_{k+1}}^{m} \circ\left(\sigma_{i}\right)_{C_{k+1}}$. This means that $f^{m^{\prime}}\left(P_{k+1}\right) \neq f^{m}\left(P_{i}\right)$. Hence $O_{f}\left(P_{k+1}\right) \cap O_{f}\left(P_{i}\right)=\varnothing$.

Set $\psi_{k+1}=\psi_{k} \circ \psi$. Then $\psi_{k+1} \circ \sigma_{k+1}=\psi_{k} \circ p_{k} \circ \iota$. By $(\mathrm{V}), a_{i} \in$ $\operatorname{Im}\left(\psi_{k+1} \circ \sigma_{k+1}\right)$. As a consequence, $P_{1}, \ldots, P_{k+1}$ satisfies $(*)_{k+1}$.

Continuing this process, we obtain a subset $S=\left\{P_{1}, P_{2}, \ldots\right\} \subset X_{f}$, a sequence $\cdots \rightarrow C_{1} \rightarrow C_{0}$ of finite morphisms of curves, and sections $\sigma_{i}: C_{i} \rightarrow X_{C_{i}}$ corresponding to $P_{i}$ for each $i \geq 1$ such that

- $\alpha_{f}\left(P_{i}\right)=\delta_{f}$ for every $i$,

- $O_{f}\left(P_{i}\right) \cap O_{f}\left(P_{j}\right)=\varnothing$ if $i \neq j$, and

- $a_{i} \in \operatorname{Im}\left(\psi_{C_{i}} \circ \sigma_{i}\right)$ for every $i$.

So it is enough to show that $S$ is a Zariski dense subset of $X$. Let $Z \subset X$ be a proper closed subset of $X$. We take a finite cover $C \rightarrow C_{0}$ such that $Z$ lifts to a proper closed subset $Z_{C} \subset X_{C}=X_{C_{0}} \times_{C_{0}} C$. Since $\psi_{C}\left(Z_{C}\right)$ is a proper closed subset of $X_{C_{0}}, a_{i} \notin \psi_{C}\left(Z_{C}\right)$ for some $i$. Take a curve $C^{\prime}$ with finite morphisms $C^{\prime} \rightarrow C$ and $C^{\prime} \rightarrow C_{i}$ which makes the following diagram commutative:

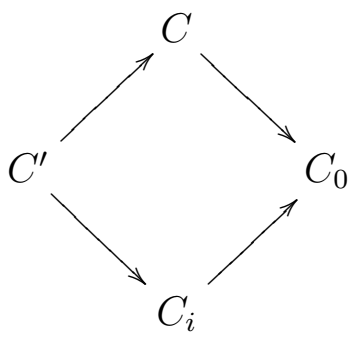

Set $Z_{C^{\prime}}=Z_{C} \times_{C} C^{\prime} \subset X_{C^{\prime}}$. Since $a_{i} \in \operatorname{Im}\left(\psi_{C^{\prime}} \circ\left(\sigma_{i}\right)_{C^{\prime}}\right)$ and $a_{i} \notin$ $\psi_{C}\left(Z_{C}\right)=\psi_{C^{\prime}}\left(Z_{C^{\prime}}\right), \operatorname{Im}\left(\left(\sigma_{i}\right)_{C^{\prime}}\right) \not \subset Z_{C^{\prime}}$. So $P_{i} \notin Z$. Therefore $S$ is a Zariski dense subset of $X$.

Theorem 7.2 includes the following result.

Corollary 7.4. Assume that $k$ is an uncountable algebraically closed field of characteristic 0 . Let $(X, f)$ be a dynamical system over $\overline{k(t)}$. Then there exists a rational point $P \in X_{f}$ such that $\alpha_{f}(P)=\delta_{f}$.

For a dynamical system on a rational variety, we can take a subset $S$ as in the statement of Theorem 7.2 over a fixed function field.

Theorem 7.5. Assume that $k$ is an uncountable algebraically closed field of characteristic 0 . Let $(X, f)$ be a dynamical system over $\overline{k(t)}$ such that $X$ is rational. Then there exists a subset $S \subset X_{f}$ such that

- There exists a function field $K$ of a curve over $k$ and a model $X_{K}$ of $X$ over $K$ such that all points in $S$ are defined over $K$, 
- $\alpha_{f}(P)=\delta_{f}$ for every $P \in S$,

- $S$ is a Zariski dense subset of $X$, and

- $O_{f}(P) \cap O_{f}(Q)=\varnothing$ if $P, Q \in S$ and $P \neq Q$.

We need a result in MSS].

Theorem 7.6 ([MSS, Theorem $3.4(\mathrm{i})]$ ). Let $f: X \rightarrow X$ and $g: Y \rightarrow-\rightarrow$ $Y$ be dominant rational self-maps on smooth projective varieties and $\phi: Y \rightarrow X$ a birational map such that $\phi \circ g=f \circ \phi$. Let $V \subset Y$ be an open subset such that $\left.\phi\right|_{V}: V \rightarrow \phi(V)$ is an isomorphism. Then $\bar{\alpha}_{g}(Q)=\bar{\alpha}_{f}(\phi(Q))$ and $\underline{\alpha}_{g}(Q)=\underline{\alpha}_{f}(\phi(Q))$ for any $Q \in Y_{g} \cap \phi^{-1}\left(X_{f}\right)$ satisfying $O_{g}(Q) \subset V$.

Proof of Theorem 7.5. Let $\phi: Y=\mathbb{P} \frac{n}{k(t)} \rightarrow X$ be a birational map. Set $g=\phi^{-1} \circ f \circ \phi$. Take open subsets $U \subset X$ and $V \subset Y$ such that $\left.\phi\right|_{V}: V \rightarrow U$ is isomorphic. We can take a curve $C$, a model $\left(X_{C} \stackrel{\mathrm{pr}_{C}}{\rightarrow} C, f_{C}\right)\left(\operatorname{resp} .\left(Y_{C}=\mathbb{P}_{k}^{n} \times C \stackrel{\mathrm{pr}_{C}}{\rightarrow} C, g_{C}\right)\right)$ of $(X, f)(\operatorname{resp} .(Y, g))$, a lift $U_{C}$ (resp. $V_{C}$ ) of $U$ (resp. $V$ ), and a birational map $\phi_{C}: Y_{C} \rightarrow X_{C}$ such that $\left.\phi_{C}\right|_{V_{C}}: V_{C} \rightarrow U_{C}$ is isomorphic.

Set $Z_{C}=Y_{C} \backslash V_{C}$. By Lemma 7.3, we can take a countable subset $M=\left\{a_{i}=\left(b_{i}, c_{i}\right)\right\}_{i=1}^{\infty} \subset V_{C}$ such that

- $M$ is Zariski dense in $Y_{C}$,

- $a_{i} \notin\left(g_{C}^{m}\right)^{-1}\left(I_{g_{C}} \cup Z_{C}\right) \cup \phi_{C}^{-1}\left(f_{C}^{m}\right)^{-1}\left(I_{f_{C}}\right)$ for every $m \geq 0$, and

- $b_{i} \notin \operatorname{pr}_{\mathbb{P}_{k}^{n}}\left(I_{g_{C}^{m}}\right)$ for every $m \geq 1$ and $i \geq 1$.

Note that $\operatorname{pr}_{\mathbb{P}_{k}^{n}}\left(I_{g_{C}^{m}}\right)$ is a proper closed subset of $\mathbb{P}_{k}^{n}$ for every $m$ because $\operatorname{dim} I_{g_{C}^{m}} \leq n-1$. For $m \geq 1$, let $J_{m} \subset \operatorname{pr}_{\mathbb{P}_{k}^{n}}\left(I_{g^{m}}\right)$ be the closed subset over which the fibers of $\left.\operatorname{pr}_{\mathbb{P}_{k}^{n}}\right|_{I_{g} m}$ have positive dimensions. Then $\operatorname{codim}_{\mathbb{P}_{k}^{n}} J_{m} \geq 2$. We take a point $q \in C$ such that $q \neq c_{i}$ for all $i$ and $\left.f_{C}\right|_{F_{q}}: F_{q} \rightarrow F_{q}$ is well-defined and dominant, where $F_{q}$ denotes the fiber of $\operatorname{pr}_{C}: Y_{C} \rightarrow C$ over $q$.

Assume that we have sections $\tau_{1}, \ldots, \tau_{k}$ of $\operatorname{pr}_{C}$ corresponding to $Q_{1}, \ldots, Q_{k} \in Y$ which satisfy the condition $(*)_{k}$ :

- $Q_{i} \in Y_{g} \cap \phi^{-1}\left(X_{f}\right)$ such that $O_{g}\left(Q_{i}\right) \subset V$ for $1 \leq i \leq k$,

- $\alpha_{g}\left(Q_{i}\right)=\delta_{g}$ for $1 \leq i \leq k$,

- $O_{g}\left(Q_{i}\right) \cap O_{g}\left(Q_{j}\right)=\varnothing$ if $1 \leq i, j \leq k$ and $i \neq j$, and

- $a_{i} \in \operatorname{Im}\left(\tau_{i}\right)$ for $1 \leq i \leq k$.

We take general hyperplanes $H_{1}, \ldots, H_{n-1}$ of $\mathbb{P}_{k}^{n}$. Then we have a line $L=H_{1} \cap \cdots \cap H_{n-1} \subset \mathbb{P}_{k}^{n}$. We can choose $H_{1}, \ldots, H_{n-1}$ as satisfying

(I) $L \not \subset \operatorname{pr}_{\mathbb{P}_{k}^{n}}\left(I_{g_{C}^{m}}\right)$ and $L \cap J_{m}=\varnothing$ for every $m \geq 1$,

(II) $L \times\{q\} \not \subset\left(\left.g_{C}^{m^{\prime}}\right|_{F_{q}}\right)^{-1}\left(\operatorname{Im}\left(g_{C}^{m} \circ \tau_{i}\right) \cap F_{q}\right)$ for every $m, m^{\prime} \geq 0$ and $1 \leq i \leq k$, and 
(III) $b_{k+1} \in L$.

Note that $\operatorname{Im}\left(g_{C}^{m} \circ \tau_{i}\right) \cap F_{q}$ is a point since $g_{C}^{m} \circ \tau_{i}$ is a section of $\operatorname{pr}_{C}$. By (I), $I_{g_{C}^{m}} \cap(L \times C) \subset Y$ is a finite set. Set $\bigcup_{m \geq 1}\left(I_{g_{C}^{m}} \cap(L \times C)\right)=$ $\left\{\left(x_{j}, y_{j}\right)\right\}_{j}$. We can construct a finite cover $\phi: C \rightarrow L$ satisfying

(1) $\phi\left(c_{k+1}\right)=b_{k+1}$,

(2) $\phi\left(y_{j}\right) \neq x_{j}$ for every $j$, and

(3) $(\phi(q), q) \notin\left(\left.g^{m^{\prime}}\right|_{F_{q}}\right)^{-1}\left(\operatorname{Im}\left(g^{m} \circ \tau_{i}\right) \cap F_{q}\right)$ for every $m, m^{\prime}$ and $1 \leq i \leq k$

by composing a fixed finite morphism $C \rightarrow L$ with a suitable automorphism on $L$.

Set $\tau_{k+1}: C \stackrel{\left(\phi, \mathrm{id}_{C}\right)}{\rightarrow} L \times C \hookrightarrow X_{C}$ and let $Q_{k+1} \in \mathbb{P}^{n}(K)$ be the corresponding rational point. Then $a_{k+1} \in \operatorname{Im}\left(\tau_{k+1}\right)$ by (1). Since $a_{k+1} \notin\left(g_{C}^{m}\right)^{-1}\left(I_{g_{C}} \cup Z_{C}\right) \cup \phi_{C}^{-1}\left(f_{C}^{m}\right)^{-1}\left(I_{f_{C}}\right), \operatorname{Im}\left(g_{C}^{m} \circ \tau_{k+1}\right) \not \subset I_{g_{C}} \cup Z_{C}$ and $\operatorname{Im}\left(f_{C}^{m} \circ \phi_{C} \circ \tau_{k+1}\right) \notin I_{f_{C}}$ for every $m \geq 0$. Hence $Q_{k+1} \in Y_{g} \cap \phi^{-1}\left(X_{f}\right)$ such that $O_{g}\left(Q_{k+1}\right) \subset V$. Further, $\operatorname{Im}\left(\tau_{k+1}\right) \cap I_{f^{m}}=\varnothing$ for every $m$ by (2). Therefore $\alpha_{g}\left(Q_{k+1}\right)$ exists and $\alpha_{g}\left(Q_{k+1}\right)=\delta_{g}$ by Theorem 6.2 (i). Moreover, $\left(g_{C}^{m^{\prime}} \circ \tau_{k+1}\right)(q) \neq\left(g^{m} \circ \tau_{i}\right)(q)$ for every $m, m^{\prime}$ and $1 \leq i \leq k$ by (3). In particular, it follows that $g_{C}^{m^{\prime}} \circ \tau_{k+1} \neq g_{C}^{m} \circ \tau_{i}$ for every $m, m^{\prime}$ and $1 \leq i \leq k$. This means that $O_{g}\left(Q_{k+1}\right) \cap O_{g}\left(Q_{i}\right)=\varnothing$ for $1 \leq i \leq k$. As a consequence, $\left\{\tau_{1}, \ldots, \tau_{k}, \tau_{k+1}\right\}$ satisfies $(*)_{k+1}$.

Continuing this process, we obtain morphisms $\tau_{1}, \tau_{2}, \ldots$ and a subset $T=\left\{Q_{1}, Q_{2}, \ldots\right\} \subset V \cap Y_{g}$. Set $P_{i}=\phi\left(Q_{i}\right)$ and $S=\left\{P_{1}, P_{2}, \ldots\right\}$. Then $P_{i}$ corresponds to a section $\sigma_{i}=\phi_{C} \circ \tau_{i}$. Since $Q_{i} \in Y_{g} \cap \phi^{-1}\left(X_{f}\right)$ and $O_{g}\left(Q_{i}\right) \subset V, \alpha_{g}\left(Q_{i}\right)=\alpha_{f}\left(P_{i}\right)$ by Theorem [7.6. So we have $\alpha_{f}\left(P_{i}\right)=\delta_{f}$. For $i, j$ with $i \neq j, O_{g}\left(Q_{i}\right) \cap O_{g}\left(Q_{j}\right)=\varnothing$ implies that $O_{f}\left(P_{i}\right) \cap O_{f}\left(P_{j}\right)=\varnothing$. Since $p_{i} \in \operatorname{Im}\left(\tau_{i}\right)$ for every $i, \bigcup_{i} \operatorname{Im}\left(\tau_{i}\right)$ is Zariski dense in $Y_{C}$ and so $\bigcup_{i} \operatorname{Im}\left(\sigma_{i}\right)$ is Zariski dense in $X_{C}$. So $S$ is Zariski dense in $X$. Therefore $S$ satisfies the claim.

\section{REFERENCES}

[Gue] V. Guedj, Ergodic properties of rational mappings with large topological degree, Ann. of Math. 161 (2005), 1589-1607.

[HiSi] M. Hindry, J. H. Silverman, Diophantine Geometry: An Introduction, Springer-Verlag, New York, 2000.

[KaSi1] S. Kawaguchi, J. H. Silverman, Examples of dynamical degree equals arithmetic degree, Michigan Math. J. 63 (2014), no. 1, 41-63.

[KaSi2] S. Kawaguchi, J. H. Silverman, On the dynamical and arithmetic degrees of rational self-maps of algebraic varieties, J. Reine Angew. Math. 713 (2016), 21-48.

[KaSi3] S. Kawaguchi, J. H. Silverman, Dynamical canonical heights for Jordan blocks, arithmetic degrees of orbits, and nef canonical heights on abelian varieties, Trans. Amer. Math. Soc. 368 (2016), no. 7, 5009-5035. 
[KoMo] J. Kollár, S. Mori, Birational geometry of algebraic varieties, Cambridge Univ. Press, 1998.

[Lan] S. Lang, Fundamentals of Diophantine Geometry, Springer-Verlag, New York, 1983.

[Mat] Y. Matsuzawa, On upper bounds of arithmetic degrees, preprint.

[MSS] Y. Matsuzawa, K. Sano, T. Shibata, Arithmetic degrees and dynamical degrees of endomorphisms on surfaces, preprint.

[Sil] J. H. Silverman, Arithmetic and dynamical degrees on abelian varieties, preprint.

[Tru] T. T. Truong, (Relative) dynamical degrees of rational maps over an algebraic closed field, preprint.

Graduate school of Mathematical Sciences, the University of Tokyo, KOMABA, TOKYO, 153-8914, JAPAN

Department of Mathematics, Graduate School of Science, Kyoto UNIVERSITY, KYOTO 606-8502, JAPAN

Department of Mathematics, Graduate School of Science, Kyoto UNIVERSITY, KYOTO 606-8502, JAPAN

E-mail address: myohsuke@ms.u-tokyo.ac.jp

E-mail address: ksano@math.kyoto-u.ac.jp

E-mail address: tshibata@math.kyoto-u.ac.jp 